\title{
Cooperative Estimation of Power and Direction of Transmission for a Directive Source
}

\author{
Sina Maleki, Philippe Ciblat, Symeon Chatzinotas, Bhavani Shankar M.R., and \\ Björn Ottersten
}

\begin{abstract}
Reliable spectrum cartography of directive sources depends on an accurate estimation of the direction of transmission (DoT) as well as the transmission power. Joint estimation of power and DoT of a directive source using ML estimation techniques is considered in this paper. We further analyze the parametric identifiability conditions of the problem, develop the estimation algorithm, and derive the Cramer-Rao-Bound (CRB) for the two situations: a) where the source signal is known to the sensors, and b) where the sensors are not aware of the source signal but its distribution. Particularly, we devise a specific sensor placement/selection setup for the symmetric antenna patterned sources which leads to identifiability of the problem. Finally, numerical results verifies the efficiency and accuracy of the provided estimation algorithms in this paper.
\end{abstract}

\section{Index Terms}

Cooperative estimation, direction of transmission (DoT), power estimation, directive source, spectrum cartography, cognitive radio.

\section{INTRODUCTION}

Database assisted dynamic resource allocation is generally considered as a technique to enable network level deployment of cognitive radios [1]-[3]. Such a database ideally should include all the required information of the incumbent network (e.g. power, location, radiation pattern,

S. Maleki, S. Chatzinotas, B. Shankar and B. Ottersten are with Interdisciplinary Centre for Security, Reliability and Trust (SnT), University of Luxembourg, L-2721, Luxembourg (e-mail: \{sina.maleki, symeon.chatzinotas, bhavani.shankar, bjorn.ottersten\}@uni.lu). P. Ciblat is with the Telecom ParisTech, Paris, France. This work is supported in part by the National Research Fund, Luxembourg under the projects SATSENT and SeMIGod and the European FP7 Project CoRaSat and H2020 SANSA. Parts of this paper have been presented in IEEE Asilomar 2015 and SAM 2016. 
bandwidth, direction of transmission, etc.) for the cognitive system intending to share the same spectrum as incumbent users, to be able to adapt its transmission parameters to the environment, without hindering operation of incumbent users. Most of the databases are obtained by collecting information from the regulatory bodies. However, such information are either not complete, or becomes outdated after a short time. This calls for a dynamic technique in order to complete the information of databases, update the existing information, or even produce a database where such information can not be obtained from regulatory bodies. Spectrum cartography or radio environment mapping is proposed as an efficient technique to produce the dynamic database of the incumbent or primary users, [4]-[8]. However, spectrum cartography can have plethora of other applications, e.g. network monitoring, malicious user detection, interference monitoring, and etc. The cornerstone of any spectrum cartography technique is a collaboration of sensors to estimate source parameters, e.g. location and power [9]-[15]. The authors of [9] employ sparse signal processing techniques to localize and estimate the power of multiple incumbent transmitters. In [10], quantized measurements are used to reduce the communications overhead and overcome the hardware complexities. And, location of incumbent users are determined in [11] assuming a fading channel model. Most of these works provide efficient tools for spectrum cartography of omni-directional sources which can be a valid assumption for lower parts of the frequency spectrum. However, considering the highly directive nature of wireless communications in higher parts of spectrum (e.g. Ka band, mmWave, etc. [16]), estimation of direction of transmission (DoT) becomes an essential component of spectrum cartography in order to obtain accurate results. For example, terrestrial microwave links in Ka band often used for mobile backhauling are highly directive, and thus for the cognitive systems such as fixed satellite services to coexist with the terrestrial links, it is important to know in which directions, the terrestrial links are operating [3], [16]. The same holds when a new terrestrial system intends to reuse the frequency of currently in use microwave links, e.g. for smart backhauling [17]. In such cases, the cognitive system needs to have a good estimate of the amount of power in a specific place in order to operate properly, and determine its transmission parameters such as carrier, power, etc., [3]. Even if the cognitive system is aware of all the underlying parameters, e.g. source power, location, etc., but still the knowledge of DoT is essential. Otherwise, the cognitive system is not able to obtain an accurate estimate of the power distribution in the environment, and either may hinder the operation of incumbent users or adapt transmission 
parameters which are not efficient.

There are few works which touch the problem of DoT estimation for spectrum cartography. An extensive set of measurements over different distances and positions is collected in [12] in order to estimate the DoT. The authors of [13] propose exhaustive search over multiple dimensions and large number of sensors to estimate the DoT. Further, the developed techniques only consider the case with Gaussian shaped antenna radiation patterns. In [18], we developed a joint power and DoT estimation for a directive source, considering the source signal to be known to the sensors. The developed algorithm of [18] can be applied to any antenna radiation pattern with a single main lobe. However, in most cases the source signal is not known, and further the algorithm of [18] incurs a high complexity in terms of synchronization between the sensors and the source, and among the sensors.

Including and in addition to the known signal model in [18], here, the joint estimation of power and DoT is also investigated by considering the source signal to be unknown but random with a known distribution. A number of sensors collect observations, and transmit their observations to a fusion center (FC). Unlike the setup in [18], the sensors are not synchronized in sampling. The FC is responsible to infer the received data and globally estimate the power and DoT.

Specifically, our main contributions in this paper are as follows:

- First we formulate and develop the required maximum likelihood (ML) estimation algorithms for the joint power and DoT estimation of a single directive source with a general single main lobe radiation pattern. On top of the known signal model considered in [18], we consider a scenario where the exact source signals are not known, but are i.i.d. randomly distributed modeled by a zero-mean Gaussian distribution. It is shown that for both known and unknown signal models, both power and DoT can be determined by a bounded linesearch over DoT.

- In addition to the algorithmic developments, we investigate the identifiability of the underlying parameter model irrespective of a specific signal model. We find a set of sufficient conditions for the identifiability. And particularly, we devise a specific sensor selection/placement setup which makes the model parameter identifiable for the symmetric antenna patterned sources.

- We derive the Cramer-Rao-Bound (CRB) of the underlying algorithms for both known and unknown signal models as the performance bounds. Further, we prove that the developed 
algorithms are unbiased and consistent, and thus converge to the true values of power and DoT for large number of samples.

- Finally, we provide a set of numerical results which verifies the efficiency of the developed algorithms, and the propositions of the paper.

The remainder of the paper is organized as follows. Following the introduction of the signal model, the underlying parameter identifiability conditions of the model are derived in Section II. Afterward, we develop the estimation algorithms by employing ML estimation techniques for both known and unknown signal models, in Sections III, and IV. Furthermore, to achieve a theoretical benchmark for performance comparison, we derive the Cramer-Rao-Bound (CRB) in these section. As shall be shown in Section V, where a set of simulations results are depicted, the developed algorithm performs close to the CRB. And finally, we draw our conclusions in Section VI.

\section{System Model And Problem Statement}

We consider a source which employs a directive antenna with a known radiation pattern, and a single main lobe (e.g. the parabolic antenna in Fig. 1). The transmission occurs in a deterministic but unknown direction. The direction of transmission (DoT) is denoted by angle $\phi$ towards a specific reference line and represents the direction of the main lobe. We denote $P_{s}$ as the source transmission power, and $M>1$ as the number of sensors which are located at different angles towards the reference line denoted by $\theta_{i}, i=1, \cdots, M$. We assume the sensors employ omni-directional antennas for signal reception. A schematic plan for the considered model of the source and the sensors is depicted in Fig. 2. We assume the observations are then sent sequentially (and orthogonally) to the FC for global data fusion (however, as shall be shown later, this can be simplified significantly by some pre-processing at the sensor level, and transmitting e.g. the energy of samples instead of each sample individually). We consider a scenario where the FC is aware of the sensors locations as well as the location of the source (and thus the angles $\theta_{i}, i=1, \cdots, M$ ). In this paper, we consider a 2 dimensional (2D) location and radiation pattern model, nevertheless the extension to the $3 \mathrm{D}$ model is straightforward. The location information can be obtained either through a database or estimated using localization techniques, e.g. [22]-[24], a priori. However, the FC is not aware of the transmission power $P_{s}$ and DoT $\phi$. The goal of the FC is to jointly estimate $P_{s}$ and $\phi$ based on sensors' observations. 
Further, we assume that the location of sensors and the source are fixed during the estimation period.

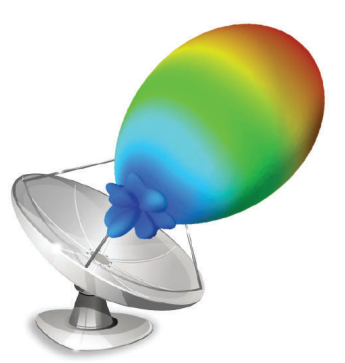

Fig. 1. A parabolic antenna with its radiation pattern as an example of a directive source.

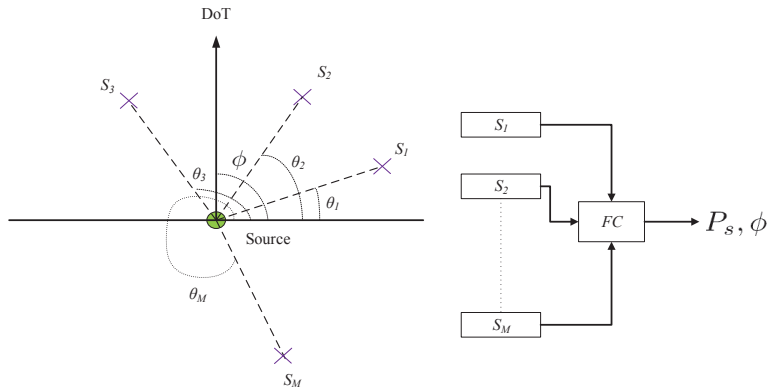

Fig. 2. Schematic plan of the considered model for the source and the sensors.

Denoting $x_{i}[n], i=1, \cdots, M$ to be the received signal at time $n$ and sensor $i$, following an additive-white-Gaussian-noise (AWGN) channel model, we have

$$
x_{i}[n]=\sqrt{P_{s} G\left(\phi, \theta_{i}\right) h\left(d_{i}\right)} s_{i}[n]+w_{i}[n],
$$

where

- $G\left(\phi, \theta_{i}\right)$ is the antenna gain in the direction of sensor $i$ known a-priori,

- $d_{i}$ is the distance between the source and the $i$-th sensor, and $h\left(d_{i}\right)$ is the path-loss,

- $s_{i}[n]$ is the source signal received at sensor $i$ at its $n$-th sampling instance,

- and $w_{i}[n]$ is the i.i.d. additive-white-Gaussian-noise (AWGN) with zero-mean and variance $\sigma_{w}^{2}$.

The path-loss is obtained by $h\left(d_{i}\right)=\left(4 \pi d_{i} / \lambda\right)^{-\gamma}, d_{i} \neq 0$, where $\lambda$ is the source signal wavelength, and $\gamma$ is the path-loss exponent. Note, this channel model does not represent the instantaneous channel variations in wireless communications, but provides a good approximation of the large-scale attenuation. For the sake of simplicity, we consider real-valued signals, $s_{i}[n], n=1, \cdots, N, i=1, \cdots, M$, however as the channel gains $h\left(d_{i}\right), i=1, \cdots, M$ are real, extension of the developed techniques in this paper to the case of complex signals is straightforward. The signal $s_{i}[n]$ is usually unknown, therefore, one way of modeling $s_{i}[n]$ is to model it as a random variable following a zero mean i.i.d. Gaussian distribution with variance $\sigma_{s}^{2}$. In this case, we further consider a case where the sensors observation sampling is asynchronous, 
which explains the subscript $i$, and this way considering enough separation between the sensors, the sensors observations become independent from each other. However, in case the sensors are synchronous in sampling, i.e. receiving the same signals from the source, the observations become correlated and this needs to be taken into account in designing the algorithms. Nevertheless, in some cases, the sensors may have knowledge about specific part of the transmitted signal, e.g. the training sequence of the communications system. In such a case, $s[n]$ is known and thus can be modeled by a deterministic signal. Here, sensors need to synchronize with the source, and further $s_{i}[n]=s[n], i=1, \cdots, M$. As in the previous model, for known signal model, the sensors observations are independent. Considering these two possible models for $s_{i}[n]$, in this paper we define the problem for a known signal (i.e. deterministic), and an unknown signal (i.e. random).

We formulate the underlying estimation problem based on ML techniques, which are widely considered as statistically efficient techniques to estimate the deterministic parameters [29]. However, before going through the detail of the estimation problem and its corresponding algorithm, in the following theorem, we establish the sufficient conditions for the considered model to be parametrically identifiable. In this theorem, $\forall$ denotes "for all", and $\exists$ denotes "there is".

Theorem 1. The model in (1) is identifiable, if the following conditions are satisfied,

1) $\forall \phi \neq \phi^{t}: \exists \theta_{i}: G\left(\phi, \theta_{i}\right) \neq G\left(\phi^{t}, \theta_{i}\right)$.

2) $\forall \Delta \neq 1$ and $\phi \neq \phi^{t}: \exists \theta_{i}: G\left(\phi, \theta_{i}\right) \neq \frac{1}{\Delta} G\left(\phi^{t}, \theta_{i}\right)$, where $\Delta=\frac{P_{s}}{P_{s}^{t}}$.

With $\phi^{t}$ and $P_{s}^{t}$ denoting the true DoT and $P_{s}$, respectively.

Proof. The proof is provided in Appendix A.

From Theorem 1, we can see that the parameter identifiability of (1) depends on the proper selection of the sensors, which in turn depends on the specific $G\left(\phi, \theta_{i}\right)$ function of the source. Below, we outline the proper selection/placement of the sensors for the specific case of symmetric antenna patterns (e.g. Horn antennas) in order to gain additional insight into the conditions outlined in Theorem 1.

In the symmetric antenna patterns, the gain function only depends on $\left|\phi-\theta_{i}\right|$ where $|\cdot|$ denotes the absolute value, and thus $G\left(\phi, \theta_{i}\right)=G\left(\phi-\theta_{i}\right)=G\left(\theta_{i}-\phi\right)=G\left(\phi-\theta_{i}+\pi\right), 0 \leq \phi \leq 2 \pi$. Note, for this discussion, we consider a symmetric antenna pattern which is a one-to-one monotonically decreasing function over $\left|\phi-\theta_{i}\right| \in[0, \pi]$, e.g. Fig. 3a. Since, we are not aware of the specific 


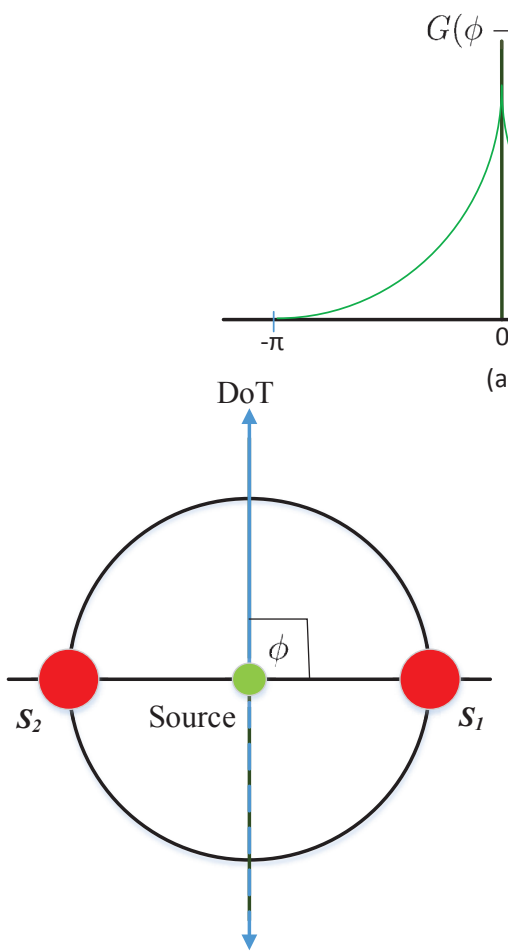

(b) (a)

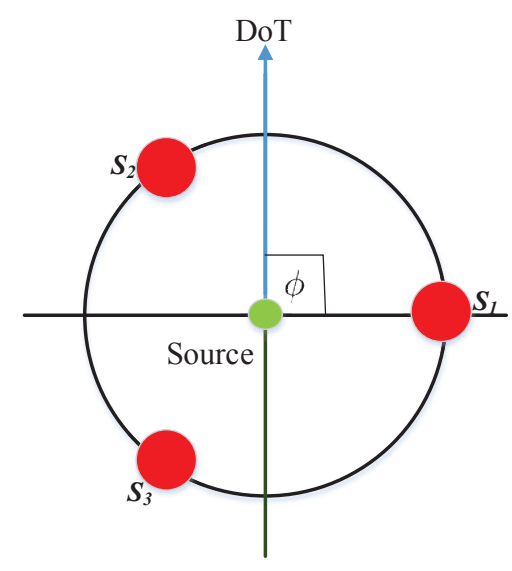

(c)

Fig. 3. (a): A symmetric antenna pattern example, (b) and (c): a not identifiable and an identifiable setup example with $\phi=\frac{\pi}{2}$ in both, and $\theta_{1}=0, \theta_{2}=\pi$ in (b), and $\theta_{1}=0, \theta_{2}=\frac{2 \pi}{3}, \theta_{3}=\frac{4 \pi}{3}$ in (c). The solid blue line shows the true DoT, and the dashed blue line in (b) depicts the ambiguity. It is clear that in (b) both $\phi=\frac{\pi}{2}$ and $\phi=\frac{3 \pi}{2}$ leads to the same power and gain product, thus the problem is not identifiable. This ambiguity is resolved in (c), because of addition of one more sensor.

value of $\left(P_{s}^{t}, \phi^{t}\right)$, we need to select the sensors such that irrespective of $\phi^{t}$, the identifiability conditions in Theorem 1 always hold.

For the first condition in Theorem 1, assuming $P_{s}^{t}$ to be known, it is easy to show that this condition is satisfied, if at least three of the sensors are located on either side of $\phi^{t}$ (e.g. Fig. 3c). Note that two sensors located on either side of $\phi^{t}$ is not sufficient for identifiability as in Fig. 3b. Further, in order to make sure that irrespective of $\phi^{t}$, the selected sensors $(M \geq 3)$ make the problem identifiable, one of the possibilities is to choose/place the sensors at equal angular separation to each other, e.g. $\theta_{i}=(i-1) \frac{2 \pi}{M}$ as in Fig. 3c.

To satisfy the second condition in Theorem 1, one approach could be to select the sensors such that $\forall \phi^{t}, \Delta \neq 1: \exists \theta_{i}: \frac{\partial G\left(\phi^{t}, \theta_{i}\right)}{\partial \phi^{t}} \neq \frac{1}{\Delta}$. Assuming a non-linear gain pattern as in Fig. 3a (which is mostly the case), again, one approach can be to select/place the sensors such that 
$\theta_{i}=(i-1) \frac{2 \pi}{M}$ (e.g. Fig. 3c). In this case, for all possible $\phi^{t}$ and $\Delta$, there is always at least one sensor $i$ for which $\frac{\partial G\left(\phi^{t}, \theta_{i}\right)}{\partial \phi^{t}} \neq \frac{1}{\Delta}$. This is an important result for identifiable estimation setup of symmetric antenna patterned sources. Hence, we highlight a generalized description of this discussion in the following proposition.

Proposition 1: If the source is equipped with a non-linear symmetric antenna pattern which is a one-to-one non-linear decreasing function over $\left|\phi-\theta_{i}\right| \in[0, \omega]$, the model parameters are identifiable if $\theta_{i}=(i-1) \frac{2 \pi}{M}, i=1, \cdots, M$, with $M>\frac{2 \pi}{\omega}$, and $\omega \leq \pi$.

Proof. The proof follows the same discussion as above and therefore is omitted.

In the following sections, we present the likelihood function of $x_{i}[n]$ for both signal models, and provide the required algorithms in the FC to estimate the power and DoT of the source using maximum likelihood (ML) estimation technique assuming the model to be identifiable.

\section{Analysis And Problem Formulation: KnOwn Signal}

\section{A. ML Estimation Problem Formulation}

Assuming $s[n]$ to be known with $\mathbb{E}\left[s^{2}[n]\right]=1$ (where $\mathbb{E}[\cdot]$ denotes the expectation), $x_{i}[n]$ is an i.i.d. real-valued random Gaussian variable with mean value of $\sqrt{P_{s} G\left(\phi, \theta_{i}\right) h\left(d_{i}\right)} s[n]$ and variance $\sigma_{w}^{2}$. Therefore, the probability density function (pdf) of the received signal at sensor $i$ and time $n$ denoted by $P\left(x_{i}[n]\right)$ becomes

$$
P\left(x_{i}[n] \mid P_{s}, \phi\right)=\frac{1}{\sqrt{2 \pi \sigma_{w}^{2}}} \exp \left\{-\frac{\left(x_{i}[n]-\sqrt{P_{s} G\left(\phi, \theta_{i}\right) h\left(d_{i}\right)} s[n]\right)^{2}}{2 \sigma_{w}^{2}}\right\} .
$$

As mentioned before, we consider a scenario where all the sensors send their observations to the FC. Then the FC estimate the power and the DoT using maximum likelihood (ML) estimation. Denoting $N$ to be total number of samples per sensor, the joint likelihood function denoted by $L$ is obtained by

$$
L\left(P_{s}, \phi\right)=\prod_{i=1}^{M} \prod_{n=1}^{N} P\left(x_{i}[n] \mid P_{s}, \phi\right),
$$

and thus after some simplifications, the log-likelihood (LL) function becomes

$$
L L\left(P_{s}, \phi\right)=M N \log \frac{1}{\sqrt{2 \pi \sigma_{w}^{2}}}-\frac{1}{2 \sigma_{w}^{2}}\left[\sum_{i=1}^{M} \sum_{n=1}^{N}\left(x_{i}[n]-\sqrt{P_{s} G\left(\phi, \theta_{i}\right) h\left(d_{i}\right)} s[n]\right)^{2}\right],
$$


where $\log$ is the natural logarithm. Since $M N \log \frac{1}{\sqrt{2 \pi \sigma_{w}^{2}}}$ and $\frac{1}{2 \sigma_{w}^{2}}$ do not depend on $P_{s}$ or $\phi$, for estimation purposes, we consider a reduced version of LL function in (3) as follows

$$
L L\left(P_{s}, \phi\right)=-\left[\sum_{i=1}^{M} \sum_{n=1}^{N}\left(x_{i}[n]-\sqrt{P_{s} G\left(\phi, \theta_{i}\right) h\left(d_{i}\right)} s[n]\right)^{2}\right] .
$$

In order to estimate $P_{s}$ and $\phi$, we consider an ML estimation problem defined as

$$
\max _{P_{s}, \phi} L L\left(P_{s}, \phi\right) \text { s.t. } P_{s} \geq 0,0 \leq \phi<2 \pi .
$$

where $L L\left(P_{s}, \phi\right)$ is obtained from (4).

\section{B. Estimation Algorithm for (5)}

To find a solution algorithm for (5), first we assume that the $\phi$ is given and find the optimal $P_{s}$, and then we insert the optimal $P_{s}$ in (5) to devise the required algorithm in order to estimate $\phi$ and $P_{s}$. As shall be shown later, for a given $\phi$ denoted by $\phi_{g}$, there is a unique $P_{s}$ which maximizes (4). For $\phi_{g}$, (5) becomes

$$
\max _{P_{s}}-\left[\sum_{i=1}^{M} \sum_{n=1}^{N}\left(x_{i}[n]-\sqrt{P_{s} G\left(\phi_{g}, \theta_{i}\right) h\left(d_{i}\right)} s[n]\right)^{2}\right] \text { s.t. } P_{s} \geq 0 .
$$

Thereby, we obtain the following theorem which provides the closed form solution of (6) denoted by $P_{s}^{*}\left(\phi_{g}\right)$.

Theorem 2: The optimal solution of (6) is obtained by

- If $\sum_{i=1}^{M} R_{i} \sqrt{G\left(\phi_{g}, \theta_{i}\right) h\left(d_{i}\right)}>0$, then

$$
P_{s}^{*}\left(\phi_{g}\right)=\left(\frac{\sum_{i=1}^{M} R_{i} \sqrt{G\left(\phi_{g}, \theta_{i}\right) h\left(d_{i}\right)}}{S \sum_{i=1}^{M} G\left(\phi_{g}, \theta_{i}\right) h\left(d_{i}\right)}\right)^{2},
$$

where $R_{i}=\sum_{n=1}^{N} x_{i}[n] s[n], S=\sum_{n=1}^{N} s^{2}[n]$.

- If $\sum_{i=1}^{M} R_{i} \sqrt{G\left(\phi_{g}, \theta_{i}\right) h\left(d_{i}\right)} \leq 0$, then

$$
P_{s}^{*}\left(\phi_{g}\right)=0
$$

Proof. The proof is provided in Appendix B.

Proposition 2: The source power estimator in Theorem 1 is unbiased and consistent for $\phi_{g}=$ $\phi^{t}$.

Proof. The proof is provided in Appendix C. 
Proposition 2 guarantees that the estimator in Theorem 2 converges to the true value of $P_{s}$, if $\phi_{g}=\phi^{t}$.

We can now rewrite (5) as follows

$$
\max _{\phi} L L\left(P_{s}^{*}(\phi), \phi\right) \text { s.t. } 0 \leq \phi<2 \pi
$$

where $P_{s}^{*}(\phi)$ is the optimal $P_{s}$ coming from Theorem 2. After some simple algebraic simplifications reported in Appendix D, we obtain

$$
\max _{\phi} U\left(\sum_{i=1}^{M} R_{i} \sqrt{G\left(\phi, \theta_{i}\right) h\left(d_{i}\right)}\right) \times \frac{\left(\sum_{i=1}^{M} R_{i} \sqrt{G\left(\phi, \theta_{i}\right) h\left(d_{i}\right)}\right)^{2}}{\sum_{i=1}^{M} G\left(\phi, \theta_{i}\right) h\left(d_{i}\right)},
$$

where $U(\bullet)$ is the Heaviside function, i.e., $U(x)=1$ if $x \geq 0$, and $U(x)=0$ otherwise. This way, we can find the optimal $\phi$ denoted by $\phi^{*}$ by an exhaustive line-search over $\phi$, and consequently $P_{s}^{*}$ from Theorem 2. The joint estimation of $P_{s}$ and $\phi$ using (9) is depicted in a more clear way in Algorithm 1.

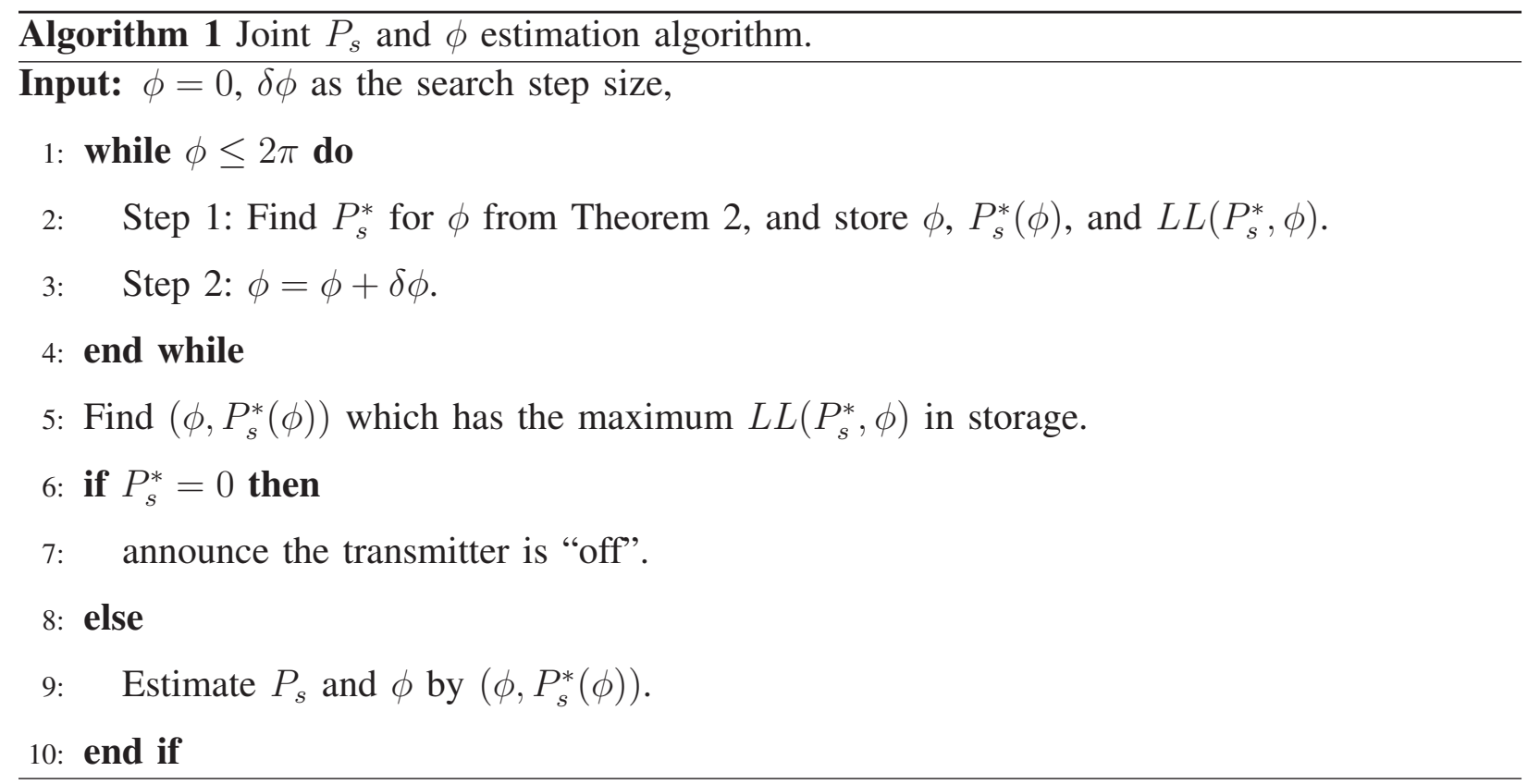

Remark 1: We can see from (9) that for the known signal scenario, the sensors only need to send $R_{i}$ to $\mathrm{FC}$ which reduces the communications overhead significantly.

Considering the fact that the computational and communication load of the FC reduces significantly by transmitting only $R_{i}$ s from the sensors, and further the fact that in each point 
of the line search over $\phi$, the corresponding power estimate is calculated by a closed form solution, the main computational complexity of the algorithm lies in the required resolution of the line-search. However, this can also be relieved significantly by performing parallel computing techniques.

Proposition 3: If the ML estimator in (9) is identifiable, the estimator in Theorem 2 is unbiased and consistent.

Proof. The proof is provided in Appendix E.

Therefore, the estimator in (9) converges to $P_{s}^{t}$ and $\phi^{t}$.

\section{CRB for Known Signal}

In order to compare the performance of the developed technique, here we obtain the Cramer-Rao-Bound (CRB) of the estimation technique developed in this paper. The CRB provides a lower-bound on the mean-square-error (MSE) of an unbiased estimator and thus $\operatorname{MSE}\left(P_{s}, \phi\right)=\operatorname{MSE}\left(P_{s}\right)+\operatorname{MSE}(\phi) \geq \operatorname{CRB}\left(P_{s}, \phi\right)=\operatorname{CRB}\left(P_{s}\right)+\operatorname{CRB}(\phi)[29]$.

Assuming that $L L\left(P_{s}, \phi\right)$ satisfies the regularity conditions, after algebraic manipulations presented in Appendix $\mathrm{F}$, we obtain the following Theorem which calculates $\operatorname{CRB}\left(P_{s}, \phi\right)$ where $G^{\prime}\left(\phi, \theta_{i}\right)=\frac{\partial G\left(\phi, \theta_{i}\right)}{\partial \phi}$.

Theorem 3: The $\mathrm{CRB}\left(P_{s}, \phi\right)$ for known signal is given by

$$
\operatorname{CRB}\left(P_{s}, \phi\right)=\frac{4 P_{s} \sigma_{w}^{2}}{N \sum_{i=1}^{M} G\left(\phi, \theta_{i}\right) h\left(d_{i}\right)}+\frac{4 \sigma_{w}^{2}}{N P_{s} \sum_{i=1}^{M} h\left(d_{i}\right) \frac{G^{\prime 2}\left(\phi, \theta_{i}\right)}{G\left(\phi, \theta_{i}\right)}},
$$

with individual $\mathrm{CRB}\left(P_{s}\right)$ and $\mathrm{CRB}(\phi)$ obtained by

$$
\begin{aligned}
& \operatorname{CRB}\left(P_{s}\right)=\frac{4 P_{s} \sigma_{w}^{2}}{N \sum_{i=1}^{M} G\left(\phi, \theta_{i}\right) h\left(d_{i}\right)}, \\
& \operatorname{CRB}(\phi)=\frac{4 \sigma_{w}^{2}}{N P_{s} \sum_{i=1}^{M} h\left(d_{i}\right) \frac{G^{\prime 2}\left(\phi, \theta_{i}\right)}{G\left(\phi, \theta_{i}\right)}} .
\end{aligned}
$$

Note that the calculation of individual CRBs is merely provided to gain more insights. Otherwise, as the estimation is jointly performed over $P_{s}$ and $\phi$, the individual CRBs can not be a good benchmark for comparison. From (10), it is clear that increasing the noise power, increases the total $\mathrm{CRB}$, but the effect of $P_{s}$ on the total $\mathrm{CRB}$ is not exactly clear. Increasing $P_{s}$ increases the $\operatorname{CRB}\left(P_{s}\right)$ but reduces the $\operatorname{CRB}(\phi)$. Additionally, increasing the number of samples reduces 
the total CRB linearly and thus the expected MSE. Furthermore, we can see that as the number of sensors increases, the CRB decreases but its effect is not linearly scaled as is the case for the number of samples $N$. Finally, it is clear that as the distance of the sensors to the source increases, CRB increases.

\section{Analysis And Problem Formulation: Unknown Signal}

\section{A. ML Estimation Problem Formulation}

In this section, ML estimation of $P_{s}$ and $\phi$ is considered for an unknown signal model $s_{i}[n]$ which follows a zero-mean normal distribution. Therefore, the probability distribution function of $x_{i}[n]$ is obtained by

$$
p\left(x_{i}[n] \mid P_{s}, \phi\right)=\frac{1}{\sqrt{2 \pi\left[P_{s} G\left(\phi, \theta_{i}\right) h\left(d_{i}\right)+\sigma_{w}^{2}\right]}} \exp \left(-\frac{1}{2} \frac{x_{i}^{2}[n]}{P_{s} G\left(\phi, \theta_{i}\right) h\left(d_{i}\right)+\sigma_{w}^{2}}\right),
$$

This way, due to the temporal and spatial independence of sensors observations, the joint likelihood of $x_{i}[n] \mathrm{s}$ becomes

$$
\begin{aligned}
L\left(P_{s}, \phi\right) & =\prod_{i=1}^{M} \prod_{n=1}^{N} p\left(x_{i}[n] \mid P_{s}, \phi\right) \\
& =\prod_{i=1}^{M} \prod_{n=1}^{N}\left(\frac{1}{\sqrt{2 \pi\left[P_{s} G\left(\phi, \theta_{i}\right) h\left(d_{i}\right)+\sigma_{w}^{2}\right]}} \exp \left(-\frac{1}{2} \frac{x_{i}^{2}[n]}{P_{s} G\left(\phi, \theta_{i}\right) h\left(d_{i}\right)+\sigma_{w}^{2}}\right)\right),
\end{aligned}
$$

To make the mathematical derivations easier, we apply the natural logarithm on both sides of (14), and thus after some simplifications, we obtain

$$
L L\left(P_{s}, \phi\right)=\sum_{i=1}^{M}-\frac{N}{2} \log \left(2 \pi\left[P_{s} G\left(\phi, \theta_{i}\right) h\left(d_{i}\right)+\sigma_{w}^{2}\right]\right)-\frac{1}{2} \frac{\sum_{n=1}^{N} x_{i}^{2}[n]}{P_{s} G\left(\phi, \theta_{i}\right) h\left(d_{i}\right)+\sigma_{w}^{2}} .
$$

As in the previous case, here we estimate $P_{s}$ and $\phi$ by maximizing the function in (15) as follows,

$$
\max _{P_{s}, \phi} L L\left(P_{s}, \phi\right) \text { s.t. } P_{s} \geq 0,0 \leq \phi \leq 2 \pi
$$

\section{B. Estimation Algorithm for (16)}

The joint estimation of $P_{s}$ and $\phi$ with the defined objective function is difficult. Therefore, first we obtain the ML of $P_{s}$ for a given $\phi$, and then we insert the obtained result in (16) in order to obtain the ML of $\phi$. 
For a given $\phi=\phi_{g}$, the optimal $P_{s}$ is obtained according to the following theorem.

Theorem 4. For a given $\phi=\phi_{g}$, the optimal $P_{s}$ denoted by $P_{s}^{*}$ is obtained by

- If $\sum_{i=1}^{M} G\left(\phi_{g}, \theta_{i}\right) h\left(d_{i}\right)\left(X_{i}-N \sigma_{w}^{2}\right) \leq 0$ then $P_{s}^{*}=0$.

- If $\sum_{i=1}^{M} G\left(\phi_{g}, \theta_{i}\right) h\left(d_{i}\right)\left(X_{i}-N \sigma_{w}^{2}\right)>0$ then $P_{s}^{*}$ is the unique solution of $\frac{\partial L L}{\partial P_{s}}=0$, with $\frac{\partial L L}{\partial P_{s}}=\sum_{i=1}^{M}-\frac{N G\left(\phi_{g}, \theta_{i}\right) h\left(d_{i}\right)}{2\left(P_{s} G\left(\phi_{g}, \theta_{i}\right) h\left(d_{i}\right)+\sigma_{w}^{2}\right)}+\frac{G\left(\phi_{g}, \theta_{i}\right) h\left(d_{i}\right) X_{i}}{2\left(P_{s} G\left(\phi_{g}, \theta_{i}\right) h\left(d_{i}\right)+\sigma_{w}^{2}\right)^{2}}$, where $X_{i}=\sum_{n=1}^{N} x_{i}^{2}[n]$.

Proof. The proof is provided in Appendix G.

Note that to find the solution of $\frac{\partial L L}{\partial P_{s}}=0$, we can either use efficient techniques such as Newton method, or exploit the quasi concavity of the LL function, and employ bisection techniques. In the latter case, we should remember to put $P_{s}^{*}=0$ in case the result of bisection technique leads to a negative power.

Proposition 4: The transmission power estimator in Theorem 4 is unbiased and consistent for $\phi_{g}=\phi^{t}$.

Proof. The proof is provided in Appendix H.

Proposition 4 guarantees that the estimator in Theorem 4 converges to $P_{s}^{t}$.

As in the case of known signal, here we insert $P_{s}^{*}$ in (15), and thus the optimal $\phi$ and consequently optimal $P_{s}$ can be estimated by solving the following line-search problem,

$$
\begin{aligned}
\max _{\phi} & \sum_{i=1}^{M}-\frac{N}{2} \log \left(2 \pi\left[P_{s}^{*}(\phi) G\left(\phi, \theta_{i}\right) h\left(d_{i}\right)+\sigma_{w}^{2}\right]\right)-\frac{1}{2} \frac{\sum_{n=1}^{N} x_{i}^{2}[n]}{P_{s}^{*}(\phi) G\left(\phi, \theta_{i}\right) h\left(d_{i}\right)+\sigma_{w}^{2}} \\
& \text { s.t. } 0 \leq \phi \leq 2 \pi,
\end{aligned}
$$

where $P_{s}^{*}(\phi)$ is obtained from Theorem 4. Since the LL function often does not have a unique global maxima in $\phi$, standard optimization algorithms such as gradient descent can lead to a local maxima which may be far away from the true $\phi$. The joint estimation of $P_{s}$ and $\phi$ using (17) is depicted in a more clear way in Algorithm 2.

Proposition 5: If the estimator in (16) is identifiable, then the estimator in (17) is asymptotically unbiased and consistent.

Proof. The proof is provided in Appendix I.

Therefore, estimator in (17) converges to $\phi^{t}$ and consequently $P_{s}^{t}$.

Remark 2: Looking at the unknown signal estimator, we can see that in this estimator, the sensors only need to communicate the accumulated energy of the received samples to the FC. 


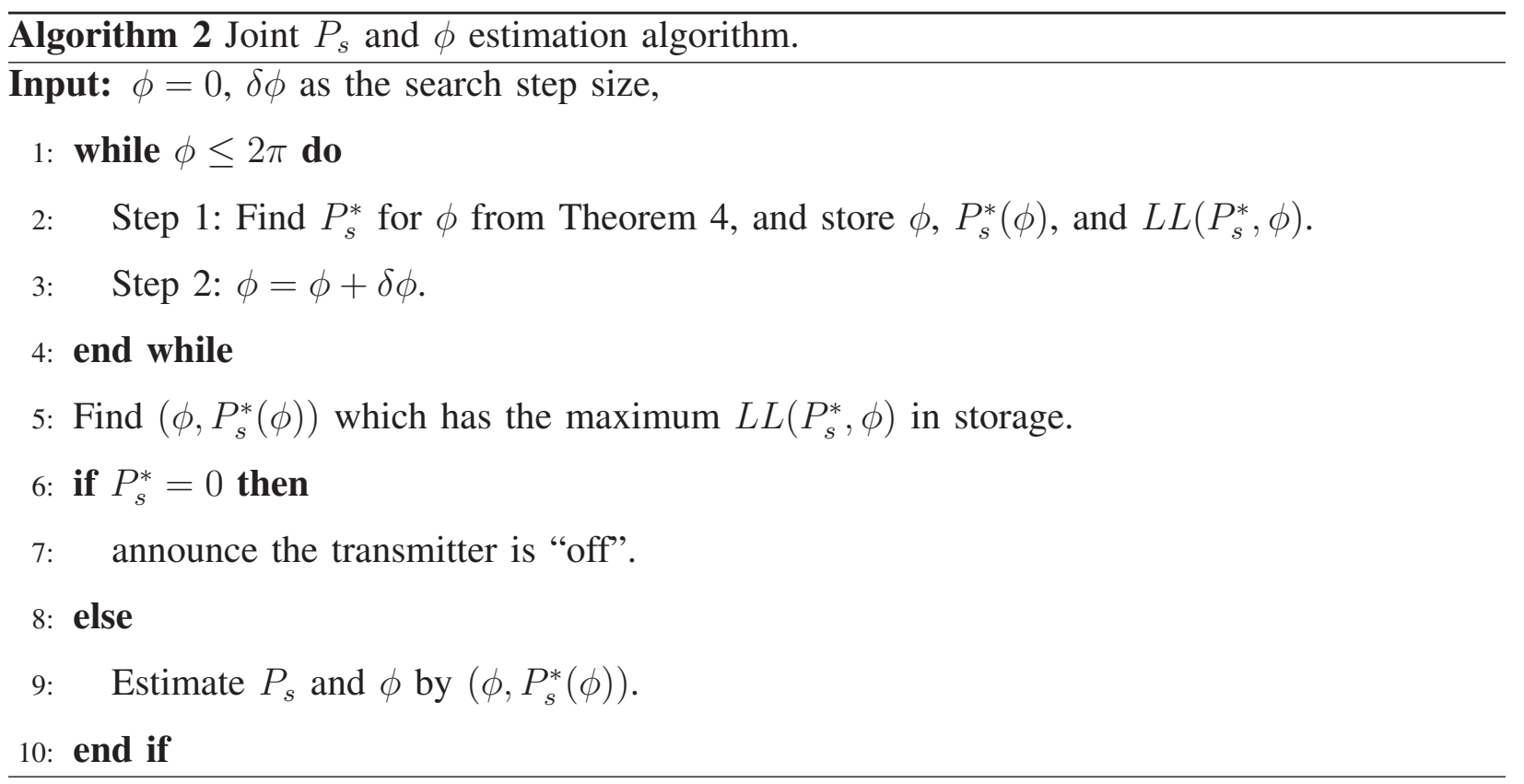

Considering the fact that the computational and communication load of the FC reduces significantly by transmitting only the accumulated energy of samples from the sensors, the main computational complexity of the algorithm lies in the required resolution of the line-search as well as finding the root of $\frac{\partial L L}{\partial P_{s}}$. However, the computational complexity induced by the line search can be relieved significantly by performing parallel computing techniques. As for the rootfinding, we can resort to fast techniques such as Newton method with quadratic convergence rate, and of low complexity. Therefore, although the complexity of algorithm in case of unknown signals may be higher than the one of known signals, but yet affordable.

\section{CRB for Unknown Signal}

As in Section III-C, after some algebraic calculations, we obtain Theorem 5, which derives the $\operatorname{CRB}\left(P_{s}, \phi\right)$ for the unknown signal scenario.

Theorem 5. We obtain $\operatorname{CRB}\left(P_{s}, \phi\right)$ for the unknown signal as follows,

$$
\operatorname{CRB}\left(P_{s}, \phi\right)=\frac{2}{N(\mathcal{A}-\mathcal{B})}\left[\sum_{i=1}^{M}\left(\frac{P_{s} h\left(d_{i}\right) G^{\prime}\left(\phi, \theta_{i}\right)}{P_{s} G\left(\phi, \theta_{i}\right) h\left(d_{i}\right)+\sigma_{w}^{2}}\right)^{2}+\sum_{i=1}^{M}\left(\frac{G\left(\phi, \theta_{i}\right) h\left(d_{i}\right)}{P_{s} G\left(\phi, \theta_{i}\right) h\left(d_{i}\right)+\sigma_{w}^{2}}\right)^{2}\right],
$$


with $G^{\prime}\left(\phi, \theta_{i}\right)=\frac{\partial G\left(\phi, \theta_{i}\right)}{\partial \phi}$, and

$$
\mathcal{A}=\sum_{i=1}^{M}\left(\frac{P_{s} h\left(d_{i}\right) G^{\prime}\left(\phi, \theta_{i}\right)}{P_{s} G\left(\phi, \theta_{i}\right) h\left(d_{i}\right)+\sigma_{w}^{2}}\right)^{2} \times \sum_{i=1}^{M}\left(\frac{G\left(\phi, \theta_{i}\right) h\left(d_{i}\right)}{P_{s} G\left(\phi, \theta_{i}\right) h\left(d_{i}\right)+\sigma_{w}^{2}}\right)^{2}
$$

and

$$
\mathcal{B}=\left(\sum_{i=1}^{M} \frac{P_{s} h^{2}\left(d_{i}\right) G\left(\phi, \theta_{i}\right) G^{\prime}\left(\phi, \theta_{i}\right)}{\left(P_{s} G\left(\phi, \theta_{i}\right) h\left(d_{i}\right)+\sigma_{w}^{2}\right)^{2}}\right)^{2}
$$

Further, the individual CRB for $P_{s}$ and $\phi$ are given by

$$
\operatorname{CRB}\left(P_{s}\right)=\frac{2}{N(\mathcal{A}-\mathcal{B})}\left[\sum_{i=1}^{M}\left(\frac{P_{s} h\left(d_{i}\right) G^{\prime}\left(\phi, \theta_{i}\right)}{P_{s} G\left(\phi, \theta_{i}\right) h\left(d_{i}\right)+\sigma_{w}^{2}}\right)^{2}\right],
$$

and

$$
\operatorname{CRB}(\phi)=\frac{2}{N(\mathcal{A}-\mathcal{B})}\left[\sum_{i=1}^{M}\left(\frac{G\left(\phi, \theta_{i}\right) h\left(d_{i}\right)}{P_{s} G\left(\phi, \theta_{i}\right) h\left(d_{i}\right)+\sigma_{w}^{2}}\right)^{2}\right] .
$$

Proof. the proof is provided in Appendix $\mathrm{J}$.

We can see that as the number of sensors $N$ increases, the nominator of $\operatorname{CRB}\left(P_{s}, \phi\right)$ increases with $N$ and denominator with $N^{2}$, and thus we can deduce that CRB decreases as $N$ increases. Opposite effect can be observed for $\sigma_{w}^{2}$, i.e. CRB increases with $\sigma_{w}^{2}$. However, the effect of the number of sensors $M, P_{s}$ and $d_{i}$ on CRB is not straightforward.

\section{Simulation Results}

In this section, our goal is to evaluate the performance of the known signal and unknown signal algorithms using some simulations results. We particularly focus on a source with a symmetric antenna pattern (with a shape similar to Fig. 3a) defined as

$$
G\left(\phi, \theta_{i}\right)= \begin{cases}100 \exp \left(-\left|\phi-\theta_{i}\right|\right) & \text { if } 0 \leq\left|\phi-\theta_{i}\right| \leq 180^{\circ} \\ 0 & \text { else. }\end{cases}
$$

This definition of antenna gain pattern matches well with most of the practical symmetric antenna patterns, e.g. Horn or parabolic antennas. Further, according to Proposition 1, we place the sensors such that $\theta_{i}=(i-1) \frac{2 \pi}{M}$ to make the setup identifiable, and without loss of generality, unless it is clearly mentioned, we assume the sensors are equally distanced from the source, and thus $\forall i: d_{i}=d$. In all the simulations, we assume DoT to be $\phi=60^{\circ}, P_{s}=0 \mathrm{dBW}$, transmit frequency denoted by $f$ to be $18 \mathrm{GHz}, \gamma=2$ (equivalent to a line-of-sight channel), and $\sigma_{w}^{2}=-136 \mathrm{dBW}$ which approximately represents the noise power of a $5 \mathrm{MHz}$ bandwidth and 
noise temperature of $T=360 \mathrm{~K}$ receiver. Note that in practice, depending on the environment, the value of $\gamma$ is often higher than 2 which is equivalent to free space path-loss. Further, the considered value of bandwidth and noise temperature in this paper does not necessarily represent a particular implementation, as the specific value of these parameters may change from one sensor technology to another, and depends on the requirement of the operators, environment and antenna technologies. Therefore, the simulations based on the chosen parameters here are provided as an academic exercise in order to illustrate the efficiency of the proposed algorithms as well as validity of claims in this paper. Before going through the detailed simulations results, please note that in all the figures, 'ks' denotes the known signal algorithm, and 'us' denotes the unknown one.

Fig. 4 depicts the normalized mean square error (NMSE) of the estimated parameters $P_{s}$ and $\phi$ with the number of samples $N$, for the known and unknown signal algorithms. In this figure, three sensors are considered for cooperative estimation setup, which are located at the distance of $d=1000 \mathrm{~m}$ to the source. The simulation result is averaged over 1000 runs and $\delta \phi=0.1$. It is clear that as $N$ increases, NMSE for both parameters and both algorithms reduces. This verifies the claims in Propositions 2 to 5. Further, in order to evaluate the performance of the algorithm with respect to those that only estimate $P_{s}$ assuming accurate $\phi$ to be known (as in e.g. [13]), the lines titled $P_{s}\left(\phi_{g}=60^{\circ}\right)$ are depicted which shows the NMSE of $P_{s}$ when $\phi$ is known for both known and unknown signal algorithms. In both cases, we can see that the NMSE in this case is extremely close to the one with estimated $\phi$.

In order to evaluate the sensitivity of the algorithms with respect to the line search step size, $\delta \phi$, in Figures 5 and 6, we depict the NMSE of $P_{s}$ and $\phi$ versus $\delta \phi$ for both known and unknown signal algorithms, respectively. In these figures, we evaluate the performance for two different values of $\phi$, i.e. $\phi=60^{\circ}, 60.5^{\circ}$. The other parameters are the same as previous scenario, with the difference of $N=1000$. An interesting trend in both figures is that for $\phi=60.5^{\circ}$ where a minimum resolution of level 0.1 is required, increasing $\delta \phi$ generally leads to an increase in NMSE. This is particularly evident for NMSE of $\phi$. However, for $\phi=60^{\circ}$, a minimum resolution of $\delta \phi=1$ is required. Here, we can see while NMSE for $\delta \phi=0.1$ is yet acceptable, however for a range of $\delta \phi$ from 1 to 6 as well as 10, the NMSE particularly for $\phi$ is very low (in our case for 1000 realizations, no error was observed). This trend can be because of the fact that here a resolution of 1 is enough and further, the gain pattern in the next step becomes largely 


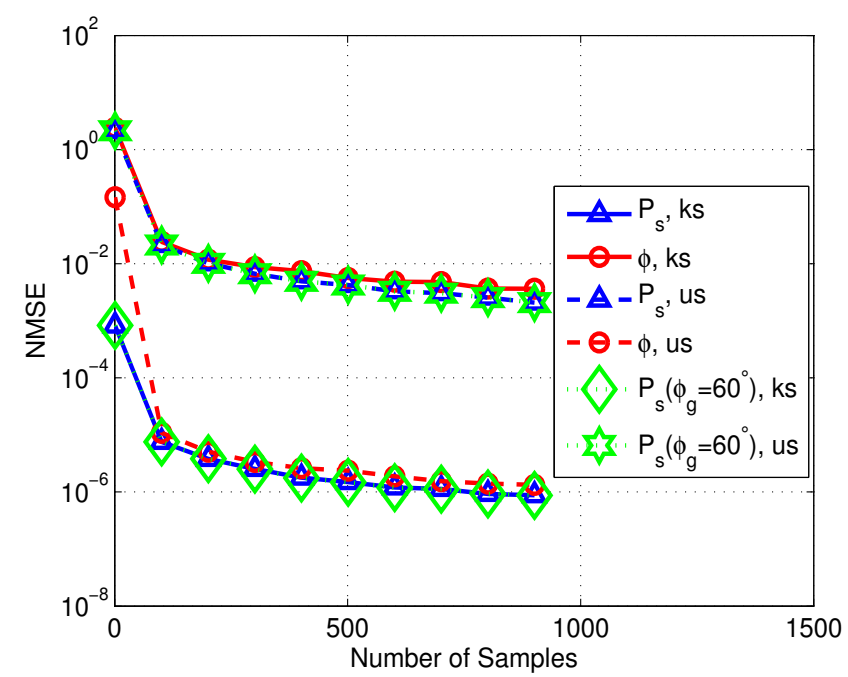

Fig. 4. NMSE of $P_{s}$ and $\phi$ versus number of samples for known and unknown signal algorithms, with $P_{s}=0 \mathrm{dBW}$, $\sigma_{w}^{2}=-136 \mathrm{dBW}, f=18 \mathrm{GHz}, \gamma=2, M=3, \theta_{i}=(i-1) \frac{360}{3}$ for $i=1,2,3$, and $d=1000 \mathrm{~m}$.

different from the previous step (something which does not usually happen for lower resolutions unless the pattern becomes very sharp), and thus a better NMSE in this case can be achieved. Nevertheless, in practice, we are mostly not aware of the minimum required step size, therefore it is more reasonable to choose a lower resolution as long as the computations are affordable. Note that in the rest of numerical results unless it is clearly mentioned we assume $\delta \phi=0.1$.

In Fig. 7, the CRB performance of the known and unknown signal algorithms is evaluated versus the number of samples for the same scenario as in Fig. 4, and for two values of $\delta \phi=0.1,1$. Here, we particularly depict the normalized total CRB (NCRB) and compared with the total NMSE as defined in Section III-C. We can see that the unknown signal estimator performs very close to $\mathrm{CRB}$ for both values of $\delta \phi$. For the known estimator, once again we can observe the importance of $\delta \phi$ in estimation accuracy. While for $\delta \phi=1$, the estimator achieves the CRB after few samples, however for $\delta \phi=0.1$, due to a higher value of estimation error in $\phi$, the performance is further away from the CRB.

After confirming the convergence of the algorithms with the number of samples in Figures 4 and 7, in Fig. 8, we intend to evaluate the effect of the distance to the source $d$, and the number of sensors $M$ on the estimation accuracy of the known signal algorithm. In this figure, we consider a configuration of 3 and 4 sensors, with the number of samples fixed at $N=1000$. 

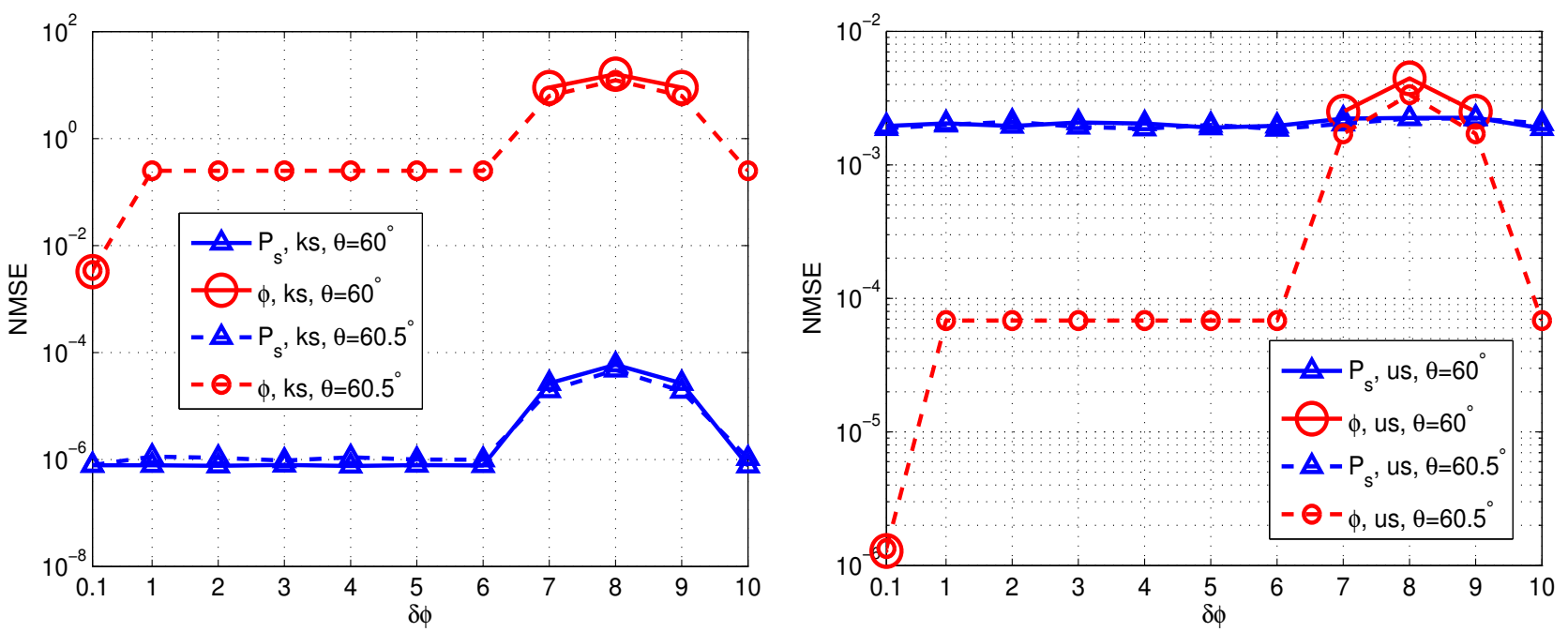

Fig. 5. NMSE of $P_{s}$ and $\phi$ versus $\delta \phi$ for known signal al-

Fig. 6. NMSE of $P_{s}$ and $\phi$ versus $\delta \phi$ for unknown signal algorithm, with $P_{s}=0 \mathrm{dBW}, \sigma_{w}^{2}=-136 \mathrm{dBW}, f=18 \mathrm{GHz}$, gorithm, with $P_{s}=0 \mathrm{dBW}, \sigma_{w}^{2}=-136 \mathrm{dBW}, f=18 \mathrm{GHz}$, $\gamma=2, M=3, \theta_{i}=(i-1) \frac{360}{3}$ for $i=1,2,3, N=1000$, and $d=1000 \mathrm{~m}$. $\gamma=2, M=3, \theta_{i}=(i-1) \frac{360}{3}$ for $i=1,2,3, N=1000$, and $d=1000 \mathrm{~m}$.

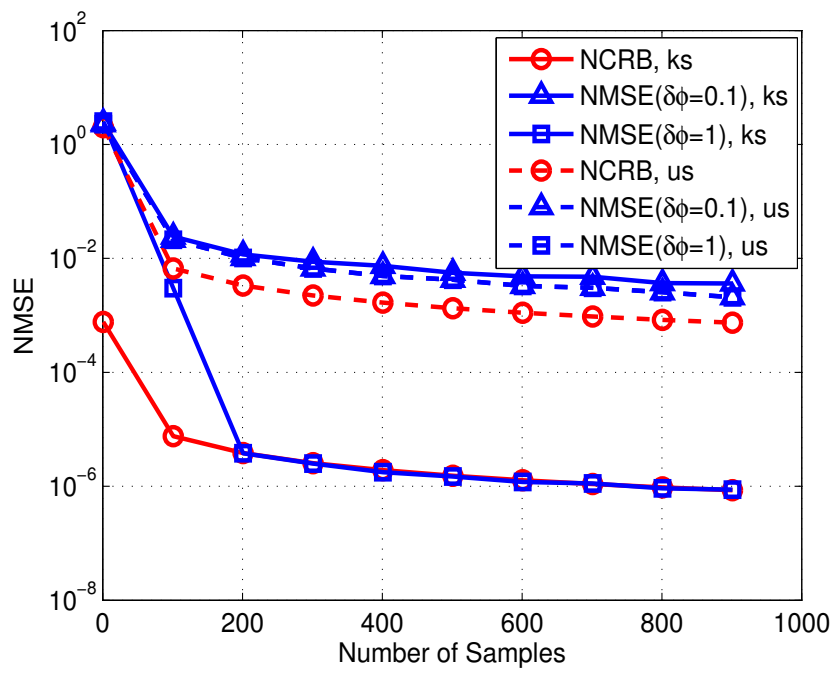

Fig. 7. NMSE and NCRB of known and unknown signal algorithms versus the number of samples, with $P_{s}=0 \mathrm{dBW}$, $\sigma_{w}^{2}=-136 \mathrm{dBW}, f=18 \mathrm{GHz}, \gamma=2, M=3, \theta_{i}=(i-1) \frac{360}{3}$ for $i=1,2,3$, and $d=1000 \mathrm{~m}$.

We can see that as $d$ increases, the estimation accuracy decreases, and the opposite effect is seen when $M$ increases, which verifies the discussion provided in Section III-C. 
In Fig. 9, the evaluation of Fig. 8 is performed for the unknown signal algorithm. In this case, the number of sensors is fixed at 3,6 and 9, and the results are averaged over 1000 runs. It is clear that increasing $d$, leads to a lower estimation accuracy for $\phi$, and increasing the number of sensors improves the estimation accuracy of $\phi$. However, in case of $P_{s}$, we have not observed a major change. Nevertheless, we have not observed the effect of number of sensors on improving estimation accuracy for all numbers of $M>3$ in our simulations. We can say if the setup with 3 sensors is spanned by the setup of higher number of sensors (e.g. 6 or 9 as in Fig. 9), the estimation accuracy may improve, however if the new setup does not include the one of 3 sensors, it may even lead to a lower estimation accuracy for $P_{s}$ based on our observations. This indeed verifies the discussion in Section IV-C, where we could not draw a definite conclusion about the effect of number of sensors on the estimation accuracy of the unknown signal algorithm.
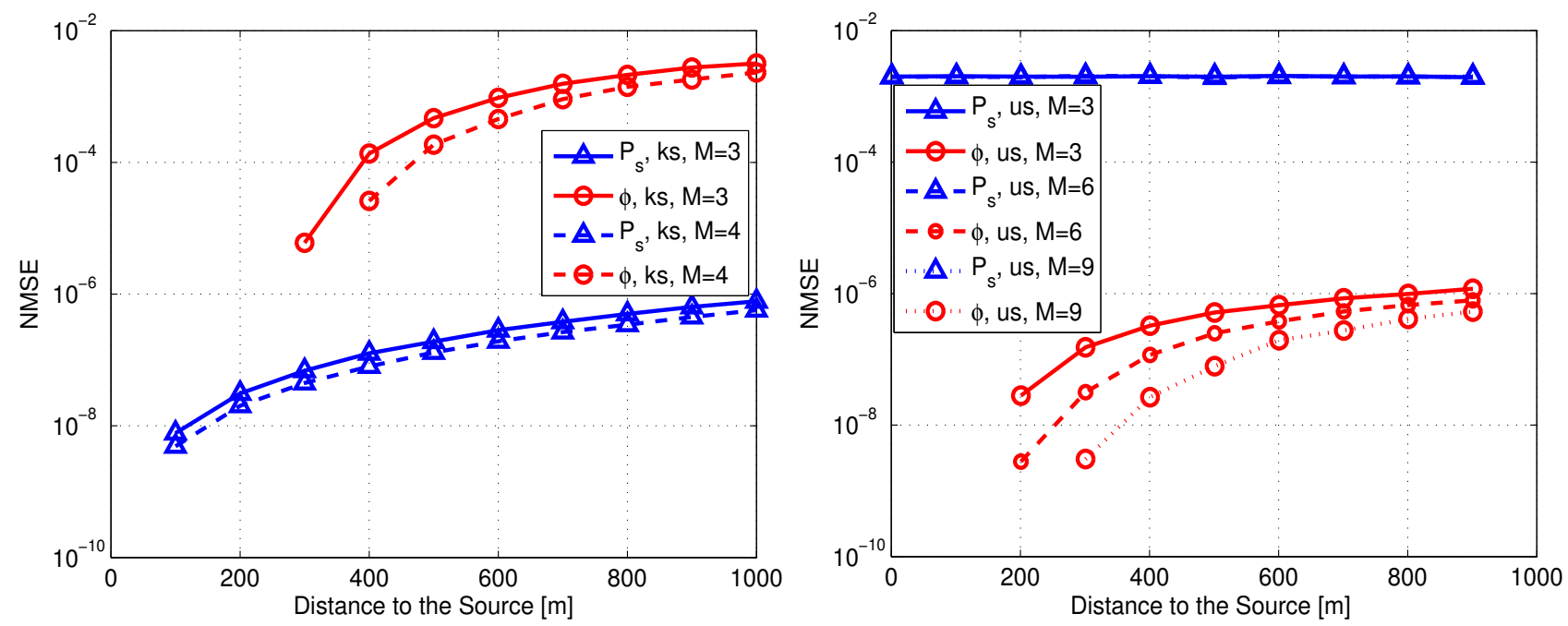

Fig. 8. NMSE of $P_{s}$ and $\phi$ versus the distance to the source for the known signal algorithm and different number of sensors, with $P_{s}=0 \mathrm{dBW}, \sigma_{w}^{2}=-136 \mathrm{dBW}, f=18 \mathrm{GHz}$, $\gamma=2, M=3,4, \theta_{i}=(i-1) \frac{360}{M}$ for $i=1, \cdots, M$, and $\mathrm{N}=1000$.
Fig. 9. NMSE of $P_{s}$ and $\phi$ versus the distance to the source for the unknown signal algorithm and different number of sensors, with $P_{s}=0 \mathrm{dBW}, \sigma_{w}^{2}=-136 \mathrm{dBW}, f=18 \mathrm{GHz}$, $\gamma=2, M=3,6,9, \theta_{i}=(i-1) \frac{360}{M}$ for $i=1, \cdots, M$, and $\mathrm{N}=1000$.

Note that so far, we assumed that the sensors are placed at equal distance to the source. In order to evaluate the performance of the system when the sensors are located at a random distance to the source, in Fig. 10, NMSE of $P_{s}$ and $\phi$ versus the number of samples is depicted for the same parameters as in Fig. 4, except for $d$, which is chosen randomly from the set 
$\{100,1000\} \mathrm{m}$. As we can see the algorithms still provide a good estimation accuracy.

After verification of the provided algorithms for the assumed radiation pattern in (21), in Fig. 11, we provide NMSE versus number of samples for the case of a more realistic antenna pattern obtained from ITU-R S.465-6 [30]. The other parameters are the same as Fig. 4. Note that in this case the antenna pattern is only a one to one function over $\left[0^{\circ}, 48^{\circ}\right]$, and thus according to Proposition 1, at least 8 sensors are required to make sure the problem is identifiable. Indeed, during the simulations, we confirmed this fact by reducing the number of sensors to 7 , and it was observed that the algorithms can not converge in this case. From the figure, we can see that the proposed algorithms provide a good estimation accuracy, and further as the number of sensors increases, the estimation accuracy clearly improves in this case.
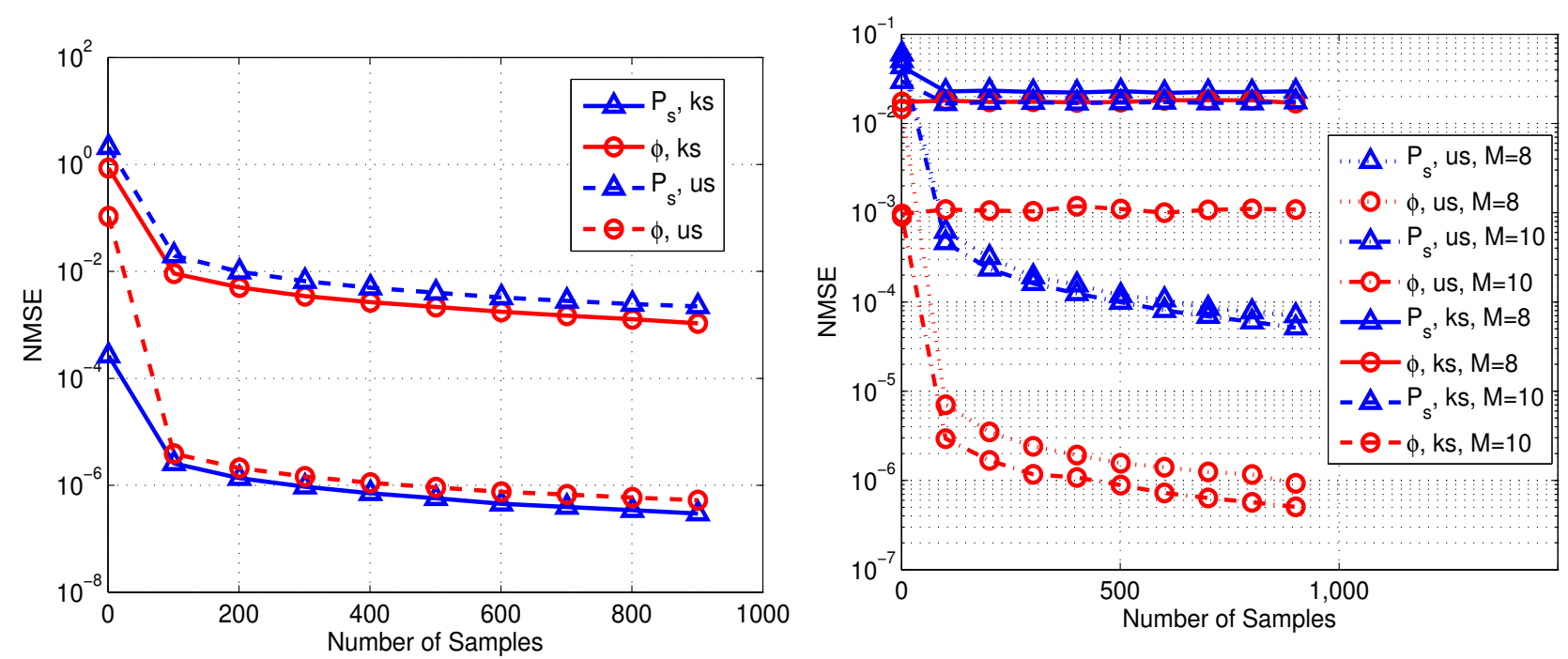

Fig. 10. NMSE of $P_{s}$ and $\phi$ versus number of samples for known and unknown signal algorithms for random $d_{i}$ s, with $P_{s}=0 \mathrm{dBW}, \sigma_{w}^{2}=-136 \mathrm{dBW}, f=18 \mathrm{GHz}, \gamma=2$, $M=3, \theta_{i}=(i-1) \frac{360}{3}$ for $i=1,2,3$.
Fig. 11. NMSE of $P_{s}$ and $\phi$ versus the number of samples for known and unknown signal algorithms, an antenna pattern based on ITU-R S.465-6, with $P_{s}=0 \mathrm{dBW}, \sigma_{w}^{2}=$ $-136 \mathrm{dBW}, f=18 \mathrm{GHz}, \gamma=2, M=8,10, \theta_{i}=(i-1) \frac{360}{M}$ for $i=1, \cdots, M$, and $d=1000$.

\section{CONCLUSiOnS AND Future WORKS}

Joint estimation of transmission power and DoT for a directive source was considered in this paper. We formulated the underlying ML estimation problems considering a known and an unknown model. The identifiability conditions for the model parameters were derived, and 
particularly we showed that for the symmetric antenna patterned sources, the sufficient conditions include a lower-bound on the number of sensors, and sensors to be placed with equal angular distances. This was followed by providing the algorithmic solution of the estimation problems which rendered to be unbiased and consistent. Further, we drove the CRB for both the known signal and unknown signal algorithms. In addition, it was shown that in case of known signal scenario, the sensors only need to transmit the cross correlation of the observation samples with the original signal, and in case of unknown signal scenario, the sensors only need to communicate the energy of the received samples. This leads to a significant reduction of communication and computation overhead.

To evaluate the performance of the developed algorithms, we performed several simulations results. It was shown that the algorithms deliver a good estimation accuracy for $P_{s}$ and $\phi$, and further their performance is close to CRB. As verified by simulations results, proper placement of the sensors according to the identifiability analysis provided in the paper is a critical parameter to consider. Another parameter which is important in obtaining accurate results is the path-loss exponent. While in the simulations results, we assumed this to be equal to 2 as in the case of free space path-loss, in reality depending on the environment this value is usually higher. Therefore, proper tuning of path-loss exponent is another parameter to take into account while calibrating the system.

In this paper, we assumed the gain pattern to be exactly known, however in practice this knowledge might not be always available or simply the antenna is not well calibrated. Development of the algorithms for unknown gain patterns is an idea of future work. Other examples of ideas for future work include better path-loss modeling, particularly using advanced wave-field estimation techniques, polarization estimation, and estimation of sources in point-tomulti-point scenarios.

\section{APPENDIX A}

\section{PROOF OF THEOREM 1}

Parameter identifiability means that model parameters can be uniquely determined from a set of noise and error free observations [27], [28]. Hence, in our case, we need to show that the set of equations $\forall i: s_{i}[n] \sqrt{P_{s} G\left(\phi, \theta_{i}\right) h\left(d_{i}\right)}=s_{i}[n] \sqrt{P_{s}^{t} G\left(\phi^{t}, \theta_{i}\right) h\left(d_{i}\right)}$ results in $P_{s}=P_{s}^{t}$ and $\phi=\phi^{t}$, with $P_{s}^{t}$ and $\phi^{t}$ denoting the true $P_{s}$ and $\phi$. Therefore, the problem boils down to finding 
the conditions under which no other $P_{s} \neq P_{s}^{t}$ or $\phi \neq \phi^{t}$ can result in $P_{s} G\left(\phi, \theta_{i}\right)=P_{s}^{t} G\left(\phi^{t}, \theta_{i}\right)$ $\forall i$.

First, we start with the case where $\phi=\phi^{t}$ but $P_{s} \neq P_{s}^{t}$. In this case, it is clear that there is no $P_{s} \neq P_{s}^{t}$ for which $P_{s} G\left(\phi, \theta_{i}\right)=P_{s}^{t} G\left(\phi^{t}, \theta_{i}\right), \forall i$. Therefore, if $\phi=\phi^{t}$, the problem is always identifiable.

Now, we consider the case where $P_{s}=P_{s}^{t}$, but $\phi \neq \phi^{t}$. This way, the problem is identifiable if $\forall i, \phi \neq \phi^{t}: G\left(\phi \neq \phi^{t}, \theta_{i}\right) \neq G\left(\phi^{t}, \theta_{i}\right)$. This condition does not hold for a general antenna pattern, all the time, e.g. symmetric antenna patterns as in Fig. 3a. In this case, the problem is identifiable if the common solution of the set $G\left(\phi, \theta_{i}\right)=G\left(\phi^{t}, \theta_{i}\right), i=1, \cdots, M$, is unique. It is clear that all the equations have at least a common solution which is $\phi=\phi^{t}$, and further, the uniqueness can be satisfied if $\forall \phi \neq \phi^{t}: \exists \theta_{i}: G\left(\phi, \theta_{i}\right) \neq G\left(\phi^{t}, \theta_{i}\right)$.

Finally, we look into the case where $P_{s} \neq P_{s}^{t}$, and $\phi \neq \phi^{t}$. Assuming $P_{s}=\Delta P_{s}^{t}$, the problem in this case is unidentifiable if $\exists \phi \neq \phi^{t}: G\left(\phi \neq \phi^{t}, \theta_{i}\right)=\frac{1}{\Delta} G\left(\phi^{t}, \theta_{i}\right), \forall i$. Therefore, the problem becomes identifiable if $\forall \Delta \neq 1, \phi \neq \phi^{t}: \exists \theta_{i}: G\left(\phi \neq \phi^{t}, \theta_{i}\right) \neq \frac{1}{\Delta} G\left(\phi^{t}, \theta_{i}\right)$. And this concludes our proof.

\section{APPENDIX B}

\section{PROOF OF THEOREM 2}

In order to find the maximum of $P_{s} \mapsto L L\left(P_{s}, \phi_{g}\right)$, we would like to analyze the shape of the function. To do that, we will calculate its derivative function. For any $P_{s} \neq 0$, we easily get

$$
\frac{\partial L L\left(P_{s}, \phi_{g}\right)}{\partial P_{s}}=\frac{1}{\sqrt{P_{s}}} \sum_{i=1}^{M} R_{i} \sqrt{G\left(\phi_{g}, \theta_{i}\right) h\left(d_{i}\right)}-S \sum_{i=1}^{M} G\left(\phi_{g}, \theta_{i}\right) h\left(d_{i}\right) .
$$

- If $\sum_{i=1}^{M} R_{i} \sqrt{G\left(\phi_{g}, \theta_{i}\right) h\left(d_{i}\right)}>0$, then the derivative function is positive as $P_{s} \rightarrow 0$. And thus the function $L L\left(\bullet, \phi_{g}\right)$ increases with $P_{s}$ until the point $P_{s}^{*}$ such that

$$
\frac{1}{\sqrt{P_{s}^{*}}} \sum_{i=1}^{M} R_{i} \sqrt{G\left(\phi_{g}, \theta_{i}\right) h\left(d_{i}\right)}=S \sum_{i=1}^{M} G\left(\phi_{g}, \theta_{i}\right) h\left(d_{i}\right) .
$$

Beyond the point $P_{s}^{*}$, the derivative function becomes negative and the function $L L\left(\bullet, \phi_{g}\right)$ decreases. Therefore the optimal point is $P_{s}^{*}$ and so we get Eq. (7).

- If $\sum_{i=1}^{M} R_{i} \sqrt{G\left(\phi_{g}, \theta_{i}\right) h\left(d_{i}\right)} \leq 0$, then the derivative function is always negative and so the function $\operatorname{LL}\left(\bullet, \phi_{g}\right)$ is monotonic decreasing in $P_{s}$. Therefore the optimal point is zero. 


\section{APPENDIX C}

\section{PROOF OF PROPOSITION 2}

We prove the proposition for the case $P_{s}^{t}>0$, the case with $P_{s}^{t}=0$ (i.e. the case where the transmitter is actually "off") can be proved in a similar way (indeed in this case for any $\phi_{g}$ including $\phi_{t}$, the estimated $P_{s}$ tends to 0 asymptotically). Denoting the true $P_{s}$ to be estimated as $P_{s}^{t}$, to prove the consistency of the estimator in Theorem 2, we need to prove that $\lim _{N \rightarrow \infty} P_{s}^{*}$ from (7) is equal to $P_{s}^{t}$. Considering the fact that $\lim _{N \rightarrow \infty} \sum_{i=1}^{M} R_{i} \sqrt{G\left(\phi^{t}, \theta_{i}\right) h\left(d_{i}\right)}=\lim _{N \rightarrow \infty} S \sqrt{P_{s}^{t}} \sum_{i=1}^{M} \sqrt{G\left(\phi^{t}, \theta_{i}\right) h\left(d_{i}\right)}>0$, we have

$$
\begin{aligned}
\lim _{N \rightarrow \infty} P_{s}^{*} & =\lim _{N \rightarrow \infty}\left(\frac{\sum_{i=1}^{M} R_{i} \sqrt{G\left(\phi^{t}, \theta_{i}\right) h\left(d_{i}\right)}}{S \sum_{i=1}^{M} G\left(\phi^{t}, \theta_{i}\right) h\left(d_{i}\right)}\right)^{2} \\
& =\lim _{N \rightarrow \infty}\left(\frac{S \sqrt{P_{s}^{t}} \sum_{i=1}^{M} G\left(\phi^{t}, \theta_{i}\right) h\left(d_{i}\right)+\sum_{i=1}^{M} \sum_{n=1}^{N} s[n] w_{i}[n] \sqrt{G\left(\phi^{t}, \theta_{i}\right) h\left(d_{i}\right)}}{S \sum_{i=1}^{M} G\left(\phi^{t}, \theta_{i}\right) h\left(d_{i}\right)}\right)^{2} \\
& =P_{s}^{t}+\lim _{N \rightarrow \infty}\left(\frac{\sum_{i=1}^{M} \sum_{n=1}^{N} s[n] w_{i}[n] \sqrt{G\left(\phi^{t}, \theta_{i}\right) h\left(d_{i}\right)}}{S \sum_{i=1}^{M} G\left(\phi^{t}, \theta_{i}\right) h\left(d_{i}\right)}\right)^{2}+\lim _{N \rightarrow \infty} \frac{2 \sqrt{P_{s}^{t}} \sum_{i=1}^{M} \sum_{n=1}^{N} s[n] w_{i}[n] \sqrt{G\left(\phi^{t}, \theta_{i}\right) h\left(d_{i}\right)}}{S \sum_{i=1}^{M} G\left(\phi^{t}, \theta_{i}\right) h\left(d_{i}\right)}, \\
& =P_{s}^{t}+0+0=P_{s}^{t},
\end{aligned}
$$

where we used the fact that $R_{i}=S \sqrt{P_{s}^{t} G\left(\phi^{t}, \theta_{i}\right) h\left(d_{i}\right)}+\sum_{n=1}^{N} s[n] w_{i}[n] \sqrt{G\left(\phi^{t}, \theta_{i}\right) h\left(d_{i}\right)}$, and $\lim _{N \rightarrow \infty} \sum_{n=1}^{N} s[n] w_{i}[n]=0$.

Further, to prove that this estimator is unbiased, we need to show that $\mathbb{E}\left(P_{s}^{*}\right)=P_{s}^{t}$. Therefore we have

$$
\begin{aligned}
\mathbb{E}\left(P_{s}^{*}\right) & =\mathbb{E}\left[\left(\frac{\sum_{i=1}^{M} R_{i} \sqrt{G\left(\phi^{t}, \theta_{i}\right) h\left(d_{i}\right)}}{S \sum_{i=1}^{M} G\left(\phi^{t}, \theta_{i}\right) h\left(d_{i}\right)}\right)^{2}\right], \\
& =\mathbb{E}\left[P_{s}^{t}+\left(\frac{\sum_{i=1}^{M} \sum_{n=1}^{N} s[n] w_{i}[n] \sqrt{G\left(\phi^{t}, \theta_{i}\right) h\left(d_{i}\right)}}{S \sum_{i=1}^{M} G\left(\phi^{t}, \theta_{i}\right) h\left(d_{i}\right)}\right)^{2}+\left(\frac{2 \sqrt{P_{s}^{t}} \sum_{i=1}^{M} \sum_{n=1}^{N} s[n] w_{i}[n] \sqrt{G\left(\phi^{t}, \theta_{i}\right) h\left(d_{i}\right)}}{S \sum_{i=1}^{M} G\left(\phi^{t}, \theta_{i}\right) h\left(d_{i}\right)}\right)\right] \\
& =P_{s}^{t}+\mathbb{E}\left[\left(\frac{\sum_{i=1}^{M} \sum_{n=1}^{N} s[n] w_{i}[n] \sqrt{G\left(\phi^{t}, \theta_{i}\right) h\left(d_{i}\right)}}{S \sum_{i=1}^{M} G\left(\phi^{t}, \theta_{i}\right) h\left(d_{i}\right)}\right)^{2}\right]+\mathbb{E}\left[\left(\frac{2 \sqrt{P_{s}^{t} \sum_{i=1}^{M} \sum_{n=1}^{N} s[n] w_{i}[n] \sqrt{G\left(\phi^{t}, \theta_{i}\right) h\left(d_{i}\right)}}}{S \sum_{i=1}^{M} G\left(\phi^{t}, \theta_{i}\right) h\left(d_{i}\right)}\right)\right], \\
& =P_{s}^{t}+0+0=P_{s}^{t},
\end{aligned}
$$

where $\mathbb{E}\left[\left(\frac{\sum_{i=1}^{M} \sum_{n=1}^{N} s[n] w_{i}[n] \sqrt{G\left(\phi^{t}, \theta_{i}\right) h\left(d_{i}\right)}}{S \sum_{i=1}^{M} G\left(\phi^{t}, \theta_{i}\right) h\left(d_{i}\right)}\right)^{2}\right]$ and $\mathbb{E}\left[\left(\frac{2 \sqrt{P_{s}^{t}} \sum_{i=1}^{M} \sum_{n=1}^{N} s[n] w_{i}[n] \sqrt{G\left(\phi^{t}, \theta_{i}\right) h\left(d_{i}\right)}}{S \sum_{i=1}^{M} G\left(\phi^{t}, \theta_{i}\right) h\left(d_{i}\right)}\right)\right]$, are found to be zero by replacing the expectation with the sample average as $n \rightarrow \infty$. And this concludes our proof. 


\section{APPENDIX D}

\section{PROOF OF EQUATION (9)}

If $\sum_{i=1}^{M} R_{i} \sqrt{G\left(\phi, \theta_{i}\right) h\left(d_{i}\right)} \geq 0$, we put (7) into (4), and obtain that we have to maximize $-\sum_{i=1}^{M} X_{i}+\frac{1}{S} \frac{\left(\sum_{i=1}^{M} R_{i} \sqrt{G\left(\phi, \theta_{i}\right) h\left(d_{i}\right)}\right)^{2}}{\sum_{i=1}^{M} G\left(\phi, \theta_{i}\right) h\left(d_{i}\right)}$ with $X_{i}=\sum_{n=1}^{N} x_{i}^{2}(n)$.

If $\sum_{i=1}^{M} R_{i} \sqrt{G\left(\phi, \theta_{i}\right) h\left(d_{i}\right)}<0, P_{s}^{*}\left(\phi_{g}\right)=0$, and so we trivially have to maximize $-\sum_{i=1}^{M} X_{i}$ which is actually constant. In this case any $\phi$ is optimal, which is not problematic in terms of spectrum cartography as $P_{s}^{*}=0$ means the source is not transmitting at this moment, therefore the direction is not important.

Consequently, we can merge both cases in a single equation as follows $-\sum_{i=1}^{M} X_{i}+$ $\delta \frac{1}{S} \frac{\left(\sum_{i=1}^{M} R_{i} \sqrt{G\left(\phi, \theta_{i}\right) h\left(d_{i}\right)}\right)^{2}}{\sum_{i=1}^{M} G\left(\phi, \theta_{i}\right) h\left(d_{i}\right)}$ with $\delta$ equal to 1 for the first case and 0 for the second case.

Moreover as $-\sum_{i=1}^{M} X_{i}$ and $S$ are independent of $\phi$, these terms can be removed and we then obtain the result provided in (9).

\section{APPENDIX E}

\section{PROOF OF PROPOSITION 3}

To prove that (9) is unbiased and consistent, it is easier to provide the same for (5). To prove consistency, it is clear that $\lim _{N \rightarrow \infty}-\left[\sum_{i=1}^{M} \sum_{n=1}^{N}\left(x_{i}[n]-\sqrt{P_{s} G\left(\phi, \theta_{i}\right) h\left(d_{i}\right)} s[n]\right)^{2}\right]=$ $\lim _{N \rightarrow \infty}-\left[\sum_{i=1}^{M} \sum_{n=1}^{N}\left(\sqrt{P_{s}^{t} G\left(\phi^{t}, \theta_{i}\right) h\left(d_{i}\right)} s[n]+w_{i}[n]-\sqrt{P_{s} G\left(\phi, \theta_{i}\right) h\left(d_{i}\right)} s[n]\right)^{2}\right]$ is maximized when $P_{s} G\left(\phi, \theta_{i}\right)=P_{s}^{t} G\left(\phi^{t}, \theta_{i}\right)$. Since the problem is assumed to be identifiable, $P_{s}=P_{s}^{t}$ and $\phi=\phi^{t}$.

To prove (5) is unbiased, we need to show that $\mathbb{E}\left(\max _{P_{s}, \phi} L L\left(P_{s}, \phi\right)\right)=\left(P_{s}^{t}, \phi^{t}\right)$. Therefore we have

$$
\begin{aligned}
\mathbb{E}\left(\max _{P_{s}, \phi} L L\left(P_{s}, \phi\right)\right) & =\mathbb{E}\left[\sum_{n=1}^{N} \max _{P_{s}, \phi}\left[-\sum_{i=1}^{M} \sum_{n=1}^{N}\left(x_{i}[n]-\sqrt{P_{s} G\left(\phi, \theta_{i}\right) h\left(d_{i}\right)} s[n]\right)^{2}\right]\right], \\
& =\mathbb{E}\left[\sum_{n=1}^{N} \min _{P_{s}, \phi}\left[\sum_{i=1}^{M} \sum_{n=1}^{N}\left(x_{i}[n]-\sqrt{P_{s} G\left(\phi, \theta_{i}\right) h\left(d_{i}\right)} s[n]\right)^{2}\right]\right],
\end{aligned}
$$
where $\min _{P_{s}, \phi}\left[\sum_{i=1}^{M} \sum_{n=1}^{N}\left(x_{i}[n]-\sqrt{P_{s} G\left(\phi, \theta_{i}\right) h\left(d_{i}\right)} s[n]\right)^{2}\right]$
$\sum_{i=1}^{M} \sum_{n=1}^{N} \min _{P_{s}, \phi}\left[\left(\left(\sqrt{P_{s}^{t} G\left(\phi^{t}, \theta_{i}\right) h\left(d_{i}\right)}-\sqrt{P_{s} G\left(\phi, \theta_{i}\right) h\left(d_{i}\right)}\right) s[n]+w_{i}[n]\right)^{2}\right]$ is similar
to minimizing variance of a non-central chi-squared distributed random variable. The 
variance of a chi-squared random variable is minimized when the non-centrality parameter becomes zero. Therefore, we obtain $P_{s} G\left(\phi, \theta_{i}\right)=P_{s}^{t} G\left(\phi^{t}, \theta_{i}\right)$, and again as the problem is assumed to be identifiable $P_{s}=P_{s}^{t}$ and $\phi=\phi^{t}$. Replacing this in (24), we obtain $\mathbb{E}\left[\max _{P_{s}, \phi} L L\left(P_{s}, \phi\right)\right]=\mathbb{E}\left[\left(P_{s}^{t}, \phi^{t}\right)\right]=\left(P_{s}^{t}, \phi^{t}\right)$. And this concludes our proof.

\section{APPENDIX F}

\section{PROOF OF THEOREM 3}

We recall that the $\mathrm{CRB}$ for parameters $\left[P_{s}, \phi\right]$ is the trace of the inverse of the Fisher Information Matrix F ( [29]) defined as

$$
\mathbf{F}=\mathbb{E}\left[\begin{array}{ll}
\frac{\partial L L}{\partial P_{s}} \frac{\partial L L}{\partial P_{s}} & \frac{\partial L L}{\partial P_{s}} \frac{\partial L L}{\partial \phi} \\
\frac{\partial L L}{\partial \phi} \frac{\partial L L}{\partial P_{s}} & \frac{\partial L L}{\partial \phi} \frac{\partial L L}{\partial \phi}
\end{array}\right]
$$

where $L L\left(P_{s}, \phi\right)$ is given by (3). After some calculations we can derive each term of the $\mathbf{F}$ matrix by $\mathbb{E}\left(\frac{\partial L L}{\partial P_{s}} \frac{\partial L L}{\partial P_{s}}\right)=\frac{N \sum_{i=1}^{M} G\left(\phi, \theta_{i}\right) h\left(d_{i}\right)}{4 P_{s} \sigma_{w}^{2}}, \mathbb{E}\left(\frac{\partial L L}{\partial \phi} \frac{\partial L L}{\partial \phi}\right)=\frac{N P_{s} \sum_{i=1}^{M} h\left(d_{i}\right) \frac{G^{\prime 2}\left(\phi, \theta_{i}\right)}{G\left(\phi, \theta_{i}\right)}}{4 \sigma_{w}^{2}}$, with $G^{\prime}\left(\phi, \theta_{i}\right)=$ $\frac{\partial G\left(\phi, \theta_{i}\right)}{\partial \phi}$, and $\mathbb{E}\left(\frac{\partial L L}{\partial P_{s}} \frac{\partial L L}{\partial \phi}\right)=\mathbb{E}\left(\frac{\partial L L}{\partial \phi} \frac{\partial L L}{\partial P_{s}}\right)=0$. This way, the inverse of $\mathbf{F}$ denoted by $\mathbf{F}^{-1}$ becomes

$$
\mathbf{F}^{-1}=\left[\begin{array}{cc}
\frac{4 P_{s} \sigma_{w}^{2}}{N \sum_{i=1}^{M} G\left(\phi, \theta_{i}\right) h\left(d_{i}\right)} & 0 \\
0 & \frac{4 \sigma_{w}^{2}}{N P_{s} \sum_{i=1}^{M} h\left(d_{i}\right) \frac{G^{\prime 2}\left(\phi, \theta_{i}\right)}{G\left(\phi, \theta_{i}\right)}}
\end{array}\right],
$$

and thus we obtain

$$
\operatorname{CRB}\left(P_{s}, \phi\right)=\operatorname{trace}\left(\mathbf{F}^{-1}\right)=\frac{4 P_{s} \sigma_{w}^{2}}{N \sum_{i=1}^{M} G\left(\phi, \theta_{i}\right) h\left(d_{i}\right)}+\frac{4 \sigma_{w}^{2}}{N P_{s} \sum_{i=1}^{M} h\left(d_{i}\right) \frac{G^{\prime 2}\left(\phi, \theta_{i}\right)}{G\left(\phi, \theta_{i}\right)}},
$$

and $\operatorname{CRB}\left(P_{s}\right)=\frac{4 P_{s} \sigma_{w}^{2}}{N \sum_{i=1}^{M} G\left(\phi, \theta_{i}\right) h\left(d_{i}\right)}, \operatorname{CRB}(\phi)=\frac{4 \sigma_{w}^{2}}{N P_{s} \sum_{i=1}^{M} h\left(d_{i}\right) \frac{G^{\prime 2}\left(\phi, \theta_{i}\right)}{G\left(\phi, \theta_{i}\right)}}$, which concludes our proof.

\section{APPENDIX G}

\section{ProOF OF THEOREM 4}

In order to prove Theorem 4, first we calculate $\frac{\partial L L\left(P_{s}, \phi_{g}\right)}{\partial P_{s}}$, and we obtain

$$
\frac{\partial L L\left(P_{s}, \phi_{g}\right)}{\partial P_{s}}=\sum_{i=1}^{M}-\frac{N G\left(\phi, \theta_{i}\right) h\left(d_{i}\right)}{2\left(P_{s} G\left(\phi, \theta_{i}\right) h\left(d_{i}\right)+\sigma_{w}^{2}\right)}+\frac{G\left(\phi, \theta_{i}\right) h\left(d_{i}\right) X_{i}}{2\left(P_{s} G\left(\phi, \theta_{i}\right) h\left(d_{i}\right)+\sigma_{w}^{2}\right)^{2}} .
$$

It is clear the the negative term in (28), i.e. $-\frac{N G\left(\phi, \theta_{i}\right) h\left(d_{i}\right)}{2\left(P_{s} G\left(\phi, \theta_{i}\right) h\left(d_{i}\right)+\sigma_{w}^{2}\right)}$ is increasing in $P_{s}$, while the positive term, i.e. $\frac{G\left(\phi, \theta_{i}\right) h\left(d_{i}\right) X_{i}}{2\left(P_{s} G\left(\phi, \theta_{i}\right) h\left(d_{i}\right)+\sigma_{w}^{2}\right)^{2}}$ is decreasing in $P_{s}$. Further, it is clear that the speed of 
the negative term growth is slower that the speed of the positive term reduction. This shows that the negative term of (28) can cut the positive term only once. For $P_{s}=0, \frac{\partial L L\left(P_{s}, \phi_{g}\right)}{\partial P_{s}}$ has two possibilities as follows.

- If $\left.\frac{\partial L L\left(P_{s}, \phi_{g}\right)}{\partial P_{s}}\right|_{P_{s}=0} \leq 0$ and thus $\sum_{i=1}^{M} G\left(\phi_{g}, \theta_{i}\right) h\left(d_{i}\right)\left(X_{i}-N \sigma_{w}^{2}\right) \leq 0$, with increasing $P_{s}$, the positive term reduces while the negative term increases, and hence $\frac{\partial L L\left(P_{s}, \phi_{g}\right)}{\partial P_{s}}$ remains not positive. Therefore the optimal $P_{s}$ in this case is $P_{s}^{*}=0$.

- If $\left.\frac{\partial L L\left(P_{s}, \phi_{g}\right)}{\partial P_{s}}\right|_{P_{s}=0}>0$ and thus $\sum_{i=1}^{M} G\left(\phi_{g}, \theta_{i}\right) h\left(d_{i}\right)\left(X_{i}-N \sigma_{w}^{2}\right)>0$, then the positive and negative terms will cut each other at $P_{s}^{*}>0$, and after that $\frac{\partial L L\left(P_{s}, \phi_{g}\right)}{\partial P_{s}}$ becomes negative. Therefore, the optimal $P_{s}$ in this case the root of $\frac{\partial L L}{\partial P_{s}}=\sum_{i=1}^{M}-\frac{N G\left(\phi, \theta_{i}\right) h\left(d_{i}\right)}{2\left(P_{s} G\left(\phi, \theta_{i}\right) h\left(d_{i}\right)+\sigma_{w}^{2}\right)}+$ $\frac{G\left(\phi, \theta_{i}\right) h\left(d_{i}\right) X_{i}}{2\left(P_{S} G\left(\phi, \theta_{i}\right) h\left(d_{i}\right)+\sigma_{w}^{2}\right)^{2}}$.

This concludes the proof, and further we can deduce that $L L\left(P_{s}, \phi_{g}\right)$ is a quasi-concave function in $P_{s}$.

\section{APPENDIX H}

\section{PROOF OF PROPOSITION 4}

As in Appendix C, first we prove Proposition 4 for $P_{s}^{t}>0$, the proof for $P_{s}^{t}=0$ is then straightforward. It is easy to show that $\lim _{N \rightarrow \infty} \sum_{i=1}^{M} G\left(\phi_{g}, \theta_{i}\right) h\left(d_{i}\right)\left(X_{i}-N \sigma^{w}\right)>0$ for $P_{s}^{t}>0$. Then, in order to prove the consistency of the estimator in Proposition 4, we need to show that the root of $\sum_{i=1}^{M}-\frac{N G\left(\phi_{g}, \theta_{i}\right) h\left(d_{i}\right)}{2\left(P_{s} G\left(\phi_{g}, \theta_{i}\right) h\left(d_{i}\right)+\sigma_{w}^{2}\right)}+\frac{G\left(\phi_{g}, \theta_{i}\right) h\left(d_{i}\right) X_{i}}{2\left(P_{s} G\left(\phi_{g}, \theta_{i}\right) h\left(d_{i}\right)+\sigma_{w}^{2}\right)^{2}}$ as $N \rightarrow \infty$ is equal to $P_{s}^{t}$. Therefore, we have

$$
\begin{aligned}
& \lim _{N \rightarrow \infty} \sum_{i=1}^{M} \frac{N G\left(\phi_{g}, \theta_{i}\right) h\left(d_{i}\right)}{2\left(P_{s} G\left(\phi_{g}, \theta_{i}\right) h\left(d_{i}\right)+\sigma_{w}^{2}\right)}=\lim _{N \rightarrow \infty} \frac{G\left(\phi_{g}, \theta_{i}\right) h\left(d_{i}\right) X_{i}}{2\left(P_{s} G\left(\phi_{g}, \theta_{i}\right) h\left(d_{i}\right)+\sigma_{w}^{2}\right)^{2}} \\
& =\lim _{N \rightarrow \infty} \frac{G\left(\phi_{g}, \theta_{i}\right) h\left(d_{i}\right)\left(P_{s}^{t} G\left(\phi_{g}, \theta_{i}\right) h\left(d_{i}\right) \sum_{n=1}^{N} s^{2}[n]+\sum_{n=1}^{N} w^{2}[n]+\sum_{n=1}^{N} \sqrt{P_{s}^{t} G\left(\phi_{g}, \theta_{i}\right) h\left(d_{i}\right)} \phi^{t} w[n]\right)}{2\left(P_{s} G\left(\phi_{g}, \theta_{i}\right) h\left(d_{i}\right)+\sigma_{w}^{2}\right)^{2}} \\
& =\lim _{N \rightarrow \infty} \frac{N G\left(\phi_{g}, \theta_{i}\right) h\left(d_{i}\right)\left(P_{s}^{t} G\left(\phi_{g}, \theta_{i}\right) h\left(d_{i}\right)+\sigma_{w}^{2}\right)}{2\left(P_{s} G\left(\phi_{g}, \theta_{i}\right) h\left(d_{i}\right)+\sigma_{w}^{2}\right)^{2}}+\lim _{N \rightarrow \infty} \frac{G\left(\phi_{g}, \theta_{i}\right) h\left(d_{i}\right)\left(\sum_{n=1}^{N} \sqrt{P_{s}^{t} G\left(\phi_{g}, \theta_{i}\right) h\left(d_{i}\right)} \phi^{t} w[n]\right)}{2\left(P_{s} G\left(\phi_{g}, \theta_{i}\right) h\left(d_{i}\right)+\sigma_{w}^{2}\right)^{2}} \\
& =\lim _{N \rightarrow \infty} \frac{N G\left(\phi_{g}, \theta_{i}\right) h\left(d_{i}\right)\left(P_{s}^{t} G\left(\phi_{g}, \theta_{i}\right) h\left(d_{i}\right)+\sigma_{w}^{2}\right)}{2\left(P_{s} G\left(\phi_{g}, \theta_{i}\right) h\left(d_{i}\right)+\sigma_{w}^{2}\right)^{2}} .
\end{aligned}
$$

We can see that by $P_{s}=P_{s}^{t}$, the above equality is valid, and as this equation has a unique root, therefore, $P_{s}=P_{s}^{t}$.

In the same way as in the case of consistency, it is easy to show that $\mathbb{E}\left(\sum_{i=1}^{M} G\left(\phi_{g}, \theta_{i}\right) h\left(d_{i}\right)\left(X_{i}-N \sigma^{w}\right)\right)>0$ for $P_{s}^{t}$. Hence, to prove that on top of consistency, the 
estimator is also unbiased, we need to show that the root of $\mathbb{E}\left(\sum_{i=1}^{M}-\frac{N G\left(\phi_{g}, \theta_{i}\right) h\left(d_{i}\right)}{2\left(P_{s} G\left(\phi_{g}, \theta_{i}\right) h\left(d_{i}\right)+\sigma_{w}^{2}\right)}+\right.$ $\left.\frac{G\left(\phi_{g}, \theta_{i}\right) h\left(d_{i}\right) X_{i}}{2\left(P_{s} G\left(\phi_{g}, \theta_{i}\right) h\left(d_{i}\right)+\sigma_{w}^{2}\right)^{2}}\right)$ is $P_{s}^{t}$. Considering the fact that $\mathbb{E}\left(X_{i}\right)=N\left(P_{s}^{t} G\left(\phi_{g}, \theta_{i}\right) h\left(d_{i}\right)+\sigma_{w}^{2}\right)$, we need to find the root of the following equation

$$
\mathbb{E}\left(\sum_{i=1}^{M}-\frac{N G\left(\phi_{g}, \theta_{i}\right) h\left(d_{i}\right)}{2\left(P_{s} G\left(\phi_{g}, \theta_{i}\right) h\left(d_{i}\right)+\sigma_{w}^{2}\right)}+\frac{N G\left(\phi_{g}, \theta_{i}\right) h\left(d_{i}\right)\left(P_{s}^{t} G\left(\phi_{g}, \theta_{i}\right) h\left(d_{i}\right)+\sigma_{w}^{2}\right)}{2\left(P_{s} G\left(\phi_{g}, \theta_{i}\right) h\left(d_{i}\right)+\sigma_{w}^{2}\right)^{2}}\right),
$$

which is clearly $P_{s}=P_{s}^{t}$, and this concludes our proof.

\section{APPENDIX I}

\section{PROOF OF PROPOSITION 5}

To prove consistency, first we try to simplify $\lim _{N \rightarrow \infty} L L\left(P_{s}, \phi\right)$. This way, we obtain

$$
\begin{array}{r}
\lim _{N \rightarrow \infty} L L\left(P_{s}, \phi\right)=\lim _{N \rightarrow \infty} \sum_{i=1}^{M}-\frac{N}{2} \log \left(2 \pi\left[P_{s} G\left(\phi, \theta_{i}\right) h\left(d_{i}\right)+\sigma_{w}^{2}\right]\right)-\frac{1}{2} \frac{\sum_{n=1}^{N} x_{i}^{2}[n]}{P_{s} G\left(\phi, \theta_{i}\right) h\left(d_{i}\right)+\sigma_{w}^{2}} \\
=\lim _{N \rightarrow \infty} \sum_{i=1}^{M}-\frac{N}{2} \log \left(2 \pi\left[P_{s} G\left(\phi, \theta_{i}\right) h\left(d_{i}\right)+\sigma_{w}^{2}\right]\right)-\frac{1}{2} \frac{N\left(P_{s}^{t} G\left(\phi^{t}, \theta_{i}\right)\right) h\left(d_{i}\right)+\sigma_{w}^{2}}{P_{s} G\left(\phi, \theta_{i}\right) h\left(d_{i}\right)+\sigma_{w}^{2}}(31)
\end{array}
$$

where we used the fact that $\lim _{N \rightarrow \infty} \frac{1}{N} \sum_{n=1}^{N} x_{i}^{2}[n]=P_{s}^{t} G\left(\phi^{t}, \theta_{i}\right) h\left(d_{i}\right)+\sigma_{w}^{2}$. Our goal is to maximize (31). Defining $A_{i}=P_{s} G\left(\phi, \theta_{i}\right) h\left(d_{i}\right)+\sigma_{w}^{2}$ and $A_{i}^{t}=P_{s}^{t} G\left(\phi^{t}, \theta_{i}\right) h\left(d_{i}\right)+\sigma_{w}^{2}$, the underlying problem becomes

$$
\max _{\substack{A_{i} \\ i=1, \cdots, M}} \sum_{i=1}^{M}\left(-\frac{N}{2}\left(2 \pi A_{i}\right)-\frac{N}{2} \frac{A_{i}^{t}}{A_{i}}\right) .
$$

It is easy to show that the solution of this equation is $\forall i: A_{i}=A_{i}^{t}$, which in turn means $\forall i$ : $P_{s} G\left(\phi, \theta_{i}\right)=P_{s}^{t} G\left(\phi^{t}, \theta_{i}\right)$. Since the problem is assumed to be identifiable, we obtain $P_{s}=P_{s}^{t}$ and $\phi=\phi^{t}$.

As in the case of consistency, to prove that the estimator is unbiased, first we obtain $\mathbb{E}\left(L L\left(P_{s}, \phi\right)\right)$ as follows,

$$
\begin{aligned}
\mathbb{E}\left(L L\left(P_{s}, \phi\right)\right) & =\mathbb{E}\left(\sum_{i=1}^{M}-\frac{N}{2} \log \left(2 \pi\left[P_{s} G\left(\phi, \theta_{i}\right) h\left(d_{i}\right)+\sigma_{w}^{2}\right]\right)-\frac{1}{2} \frac{\sum_{n=1}^{N} x_{i}^{2}[n]}{P_{s} G\left(\phi, \theta_{i}\right) h\left(d_{i}\right)+\sigma_{w}^{2}}\right) \\
= & \mathbb{E}\left(\sum_{i=1}^{M}-\frac{N}{2} \log \left(2 \pi\left[P_{s} G\left(\phi, \theta_{i}\right) h\left(d_{i}\right)+\sigma_{w}^{2}\right]\right)-\frac{N}{2} \frac{P_{s}^{t} G\left(\phi^{t}, \theta_{i}\right) h\left(d_{i}\right)+\sigma_{w}^{2}}{P_{s} G\left(\phi, \theta_{i}\right) h\left(d_{i}\right)+\sigma_{w}^{2}}\right) .
\end{aligned}
$$

Again with changing the variables to $A_{i}=P_{s} G\left(\phi, \theta_{i}\right) h\left(d_{i}\right)+\sigma_{w}^{2}$ and $A_{i}^{t}=P_{s}^{t} G\left(\phi^{t}, \theta_{i}\right) h\left(d_{i}\right)+\sigma_{w}^{2}$, we can easily show that $\forall i: A_{i}=A_{i}^{t}$ maximizes $\mathbb{E}\left(L L\left(A_{i}\right)\right)$, which in the same way as consistency, we can deduce $P_{s}=P_{s}^{t}$ and $\phi=\phi^{t}$. And this concludes our proof. 


\section{APPENDIX J}

\section{ProOF OF THEOREM 5}

As in the case of Theorem 3, here again we need to calculate the Fisher Information Matrix, $\mathbf{F}$. After some calculations, each elements of $\mathbf{F}$ can be obtained as follows,

$$
\begin{gathered}
\mathbb{E}\left(\frac{\partial L L}{\partial P_{s}} \frac{\partial L L}{\partial P_{s}}\right)=\frac{N}{2} \sum_{i=1}^{M}\left(\frac{G\left(\phi, \theta_{i}\right) h\left(d_{i}\right)}{P_{s} G\left(\phi, \theta_{i}\right) h\left(d_{i}\right)+\sigma_{w}^{2}}\right)^{2} \\
\mathbb{E}\left(\frac{\partial L L}{\partial \phi} \frac{\partial L L}{\partial \phi}\right)=\frac{N}{2} \sum_{i=1}^{M}\left(\frac{P_{s} h\left(d_{i}\right) G^{\prime}\left(\phi, \theta_{i}\right)}{P_{s} G\left(\phi, \theta_{i}\right) h\left(d_{i}\right)+\sigma_{w}^{2}}\right)^{2}, \\
\mathbb{E}\left(\frac{\partial L L}{\partial P_{s}} \frac{\partial L L}{\partial \phi}\right)=\mathbb{E}\left(\frac{\partial L L}{\partial \phi} \frac{\partial L L}{\partial P_{s}}\right)=\frac{N}{2}\left(\sum_{i=1}^{M} \frac{P_{s} h^{2}\left(d_{i}\right) G\left(\phi, \theta_{i}\right) G^{\prime}\left(\phi, \theta_{i}\right)}{\left(P_{s} G\left(\phi, \theta_{i}\right) h\left(d_{i}\right)+\sigma_{w}^{2}\right)^{2}}\right) .
\end{gathered}
$$

Calculating $\mathbf{F}^{-1}$, we obtain

$$
\mathbf{F}^{-1}=\frac{1}{\mathcal{A}-\mathcal{B}}\left[\begin{array}{cc}
\frac{N}{2} \sum_{i=1}^{M}\left(\frac{P_{s} h\left(d_{i}\right) G^{\prime}\left(\phi, \theta_{i}\right)}{P_{s} G\left(\phi, \theta_{i}\right) h\left(d_{i}\right)+\sigma_{w}^{2}}\right)^{2} & -\frac{N}{2} \sum_{i=1}^{M} \frac{P_{s} h^{2}\left(d_{i}\right) G\left(\phi, \theta_{i}\right) G^{\prime}\left(\phi, \theta_{i}\right)}{\left(P_{s} G\left(\phi, \theta_{i}\right) h\left(d_{i}\right)+\sigma_{w}^{2}\right)^{2}} \\
-\frac{N}{2} \sum_{i=1}^{M} \frac{P_{s} h^{2}\left(d_{i}\right) G\left(\phi, \theta_{i}\right) G^{\prime}\left(\phi, \theta_{i}\right)}{\left(P_{s} G\left(\phi, \theta_{i}\right) h\left(d_{i}\right)+\sigma_{w}^{2}\right)^{2}} & \frac{N}{2} \sum_{i=1}^{M}\left(\frac{G\left(\phi, \theta_{i}\right) h\left(d_{i}\right)}{P_{s} G\left(\phi, \theta_{i}\right) h\left(d_{i}\right)+\sigma_{w}^{2}}\right)^{2}
\end{array}\right],
$$

with

$$
\mathcal{A}=\frac{N}{2} \sum_{i=1}^{M}\left(\frac{P_{s} h\left(d_{i}\right) G^{\prime}\left(\phi, \theta_{i}\right)}{P_{s} G\left(\phi, \theta_{i}\right) h\left(d_{i}\right)+\sigma_{w}^{2}}\right)^{2} \times \frac{N}{2} \sum_{i=1}^{M}\left(\frac{G\left(\phi, \theta_{i}\right) h\left(d_{i}\right)}{P_{s} G\left(\phi, \theta_{i}\right) h\left(d_{i}\right)+\sigma_{w}^{2}}\right)^{2}
$$

and

$$
\mathcal{B}=\frac{N^{2}}{4}\left(\sum_{i=1}^{M} \frac{P_{s} h^{2}\left(d_{i}\right) G\left(\phi, \theta_{i}\right) G^{\prime}\left(\phi, \theta_{i}\right)}{\left(P_{s} G\left(\phi, \theta_{i}\right) h\left(d_{i}\right)+\sigma_{w}^{2}\right)^{2}}\right)^{2} .
$$

By deriving the trace of $\mathbf{F}^{-1}$, we can easily obtain $\operatorname{CRB}\left(P_{s}, \phi\right)$ for the unknown signal by

$$
\operatorname{CRB}\left(P_{s}, \phi\right)=\frac{1}{\mathcal{A}-\mathcal{B}}\left[\frac{N}{2} \sum_{i=1}^{M}\left(\frac{P_{s} h\left(d_{i}\right) G^{\prime}\left(\phi, \theta_{i}\right)}{P_{s} G\left(\phi, \theta_{i}\right) h\left(d_{i}\right)+\sigma_{w}^{2}}\right)^{2}+\frac{N}{2} \sum_{i=1}^{M}\left(\frac{G\left(\phi, \theta_{i}\right) h\left(d_{i}\right)}{P_{s} G\left(\phi, \theta_{i}\right) h\left(d_{i}\right)+\sigma_{w}^{2}}\right)^{2}\right],
$$

The individual $\mathrm{CRB}$ for $P_{s}$ and $\phi$ are then given by $\operatorname{CRB}\left(P_{s}\right)=$ $\frac{1}{\mathcal{A}-\mathcal{B}}\left[\frac{N}{2} \sum_{i=1}^{M}\left(\frac{P_{s} h\left(d_{i}\right) G^{\prime}\left(\phi, \theta_{i}\right)}{P_{s} G\left(\phi, \theta_{i}\right) h\left(d_{i}\right)+\sigma_{w}^{2}}\right)\right], \quad$ and $\operatorname{CRB}(\phi)=\frac{1}{\mathcal{A}-\mathcal{B}}\left[\frac{N}{2} \sum_{i=1}^{M}\left(\frac{G\left(\phi, \theta_{i}\right) h\left(d_{i}\right)}{P_{s} G\left(\phi, \theta_{i}\right) h\left(d_{i}\right)+\sigma_{w}^{2}}\right)^{2}\right]$, which concludes our proof. 


\section{REFERENCES}

[1] R. Doost-Mohammady, K.R. Chowdhury, "Design of spectrum database assisted cognitive radio vehicular networks," 7th International ICST Conference on Cognitive Radio Oriented Wireless Networks and Communications (CROWNCOM), Stockholm, 2012, pp. 1-5.

[2] H.R. Karimi, "Geolocation databases for white space devices in the UHF TV bands: Specification of maximum permitted emission levels," 2011 IEEE Symposium on New Frontiers in Dynamic Spectrum Access Networks (DySPAN), Aachen, 2011, pp. 443-454.

[3] E. Lagunas, S.K. Sharma, S. Maleki, S. Chatzinotas, B. Ottersten, "Resource Allocation for Cognitive Satellite Communications With Incumbent Terrestrial Networks," IEEE Transactions on Cognitive Communications and Networking, vol. 1, no. 3, pp. 305-317, Sept. 2015.

[4] H.B. Yilmaz, T. Tugcu, F. Alagoz, S. Bayhan, "Radio environment map as enabler for practical cognitive radio networks," IEEE Communications Magazine, vol.51, no.12, pp.162-169, December 2013.

[5] S. Debroy, S. Bhattacharjee, M. Chatterjee, "Spectrum Map and its application in Resource Management in Cognitive Radio Networks," to appear in IEEE Transactions on Cognitive Communications and Networking.

[6] J. Riihijarvi, P. Mahonen, "Exploiting Spatial Statistics of Primary and Secondary Users towards Improved Cognitive Radio Networks," 3rd International Conference on Cognitive Radio Oriented Wireless Networks and Communications, 2008. CrownCom 2008., 15-17 May 2008.

[7] Y. Li, T.T. Quang, Y. Kawahara, T. Asami, M. Kusunoki, "Building a spectrum map for future cognitive radio technology", Proceedings of the 2009 ACM workshop on Cognitive radio networks, September 2009.

[8] V. Atanasovski, J. van de Beek, A. Dejonghe, D. Denkovski, L. Gavrilovska, S. Grimoud, P. Mahonen, M. Pavloski, V. Rakovic, J. Riihijarvi, B. Sayrac, "Constructing radio environment maps with heterogeneous spectrum sensors," 2011 IEEE Symposium on New Frontiers in Dynamic Spectrum Access Networks (DySPAN), pp.660-661, 3-6 May 2011.

[9] J.A. Bazerque, G.B. Giannakis, "Distributed Spectrum Sensing for Cognitive Radio Networks by Exploiting Sparsity,” IEEE Transactions on Signal Processing, vol.58, no.3, pp.1847-1862, March 2010.

[10] D. Romero, S-J. Kim, R. Lopez-Valcarce, G.B. Giannakis, "Spectrum cartography using quantized observations," in 2015 IEEE International Conference on Acoustics, Speech and Signal Processing (ICASSP), pp.3252-3256, 19-24 April 2015.

[11] H.B. Yilmaz, T. Tugcu "Location estimation-based radio environment map construction in fading channels", Wirel. Commun. Mob. Comput., vol.15, pp.561-570, 2015.

[12] L. Bolea, J. Perez-Romero, R. Agusti, O. Sallent, “Context Discovery Mechanisms for Cognitive Radio," IEEE Vehicular Technology Conference (VTC Spring), pp.1-5, May 2011.

[13] R.K. Martin, R. Thomas, "Algorithms and bounds for estimating location, directionality, and environmental parameters of primary spectrum users," IEEE Transactions on Wireless Communications, vol.8, no.11, pp.5692-5701, November 2009.

[14] J. Werner, J. Wang, A. Hakkarainen, N. Gulati, D. Patron, D. Pfeil, K. Dandekar, D. Cabric, M. Valkama, "Sectorized Antenna-based DoA Estimation and Localization: Advanced Algorithms and Measurements," IEEE Journal on Selected Areas in Communications, vol.33, no.11, pp.2272-2286, Nov. 2015.

[15] J. Werner, J. Wang, A. Hakkarainen, D. Cabric, M. Valkama, "Performance and Cramer-Rao Bounds for DoA/RSS Estimation and Transmitter Localization Using Sectorized Antennas," to appear in IEEE Transactions on Vehicular Technology.

[16] S. Maleki, S. Chatzinotas, B. Evans, K. Liolis, J. Grotz, A. Vanelli-Coralli, N. Chuberre, "Cognitive Spectrum Utilization in Ka Band Multibeam Satellite Communications”, IEEE Communications Magazine, vol.53, no.3, pp.24,29, March 2015. 
[17] X. Artiga, A.I. Prez-Neira, "Shared Access Terrestrial-Satellite Backhaul Network enabled by Smart Antennas: SANSA", in Proceedings of the 2015 European Conference on Networks and Communications (EuCNC 2015), 29 June - 2 July 2015.

[18] S. Maleki, P. Ciblat, S. Chatzinotas, D. Kapetanovic, B. Ottersten, "Cooperative Power and DoT Estimation for a Directive Source", Asilomar Conference on Signals, Systems, and Computers 2015, November 2015.

[19] H. Krim, M. Viberg, “Two decades of array signal processing research: the parametric approach,” IEEE Signal Processing Magazine, vol.13, no.4, pp.67-94, July 1996.

[20] M. Viberg, and B. Ottersten, "Sensor array processing based on subspace fitting," IEEE Transactions on Signal Processing, vol.39, no.5, pp.1110-1121, May 1991.

[21] P. Stoica, A. Nehorai “MUSIC, maximum likelihood, and Cramer-Rao bound," IEEE Transactions on Acoustics, Speech and Signal Processing, vol.37, no.5, pp.720-741, May 1989.

[22] Y-D. Huang, M. Barkat, "Near-field multiple source localization by passive sensor array," IEEE Transactions on Antennas and Propagation, vol.39, no.7, pp.968-975, July 1991.

[23] J.C. Chen, R.E. Hudson, K. Yao, "Maximum-likelihood source localization and unknown sensor location estimation for wideband signals in the near-field," IEEE Transactions on Signal Processing, vol.50, no.8, pp.1843,1854, Aug 2002.

[24] E. Grosicki, K. Abed-Meraim, Y. Hua, "A weighted linear prediction method for near-field source localization," IEEE Transactions on Signal Processing, vol.53, no.10, pp.3651,3660, Oct. 2005.

[25] S. Boyd, and L. Vandenberghe, "Convex optimization”, Cambridge University Press, 2004.

[26] E.C.Y. Peh, Y-C. Liang, Y.L. Guan, Y. Zeng, "Optimization of Cooperative Sensing in Cognitive Radio Networks: A Sensing-Throughput Tradeoff View," IEEE Transactionson Vehicular Technology, vol.58, no.9, pp.5294-5299, November 2009.

[27] S. Audoly, G. Bellu, L. D’Angio, M.P. Saccomani, C. Cobelli, "Global identifiability of nonlinear models of biological systems," IEEE Transactions on Biomedical Engineering, vol.48, no.1, pp.55,65, Jan. 2001.

[28] J. Li, P. Stoica, L. Xu, W. Roberts, “On Parameter Identifiability of MIMO Radar," IEEE Signal Processing Letters, vol.14, no.12, pp.968,971, Dec. 2007.

[29] S.M. Kay, "Fundamentals of statistical signal processing, volume I: Estimation theory.", Prentice Hall PTR, 1993.

[30] ITU-R S.465-6, "Reference radiation pattern for earth station antennas in the fixed-satellite service for use in coordination and interference assessment in the frequency range from 2 to $31 \mathrm{GHz}$," Series of ITU-R Recommendations, Jan, 2010. 